\title{
Arginine-Vasopressin Expressing Neurons in the Murine Suprachiasmatic Nucleus Exhibit a Circadian Rhythm in Network Coherence in vivo
}

\author{
Adam Stowie, Zhimei Qiao, Daniella Do Carmo Buonfiglio, J. Christopher Ehlen, Morris \\ Benveniste, Alec J. Davidson \\ Morehouse School of Medicine, Atlanta GA, United States
}

\begin{abstract}
$\underline{\text { Abstract }}$
The Suprachiasmatic Nucleus (SCN) is composed of functionally distinct subpopulations of GABAergic neurons such as vasoactive intestinal polypeptide (VIP)-, arginine vasopressin (AVP)-, gastrin releasing peptide (GRP)-, and neuromedin $S$ (NMS)-expressing neurons which form a neural network responsible for synchronizing most physiological and behavioral circadian rhythms in mammals. To date, little is known regarding which aspects of SCN rhythmicity are generated by individual SCN neurons or neuronal sub-populations and which aspects result from neuronal interaction within a network. In this study, we address this question utilizing in vivo miniaturized microscopy to measure fluorescent GCaMP-mediated calcium dynamics in AVP neurons in the intact SCN of awake, behaving mice. This approach permits analysis of rhythms of single cells, populations, and correlational analysis among groups of AVP neurons in a field of view across the circadian and diurnal day and night. We report that AVP neurons in the murine SCN exhibit a periodic oscillatory increase in calcium of approximately 14 seconds across the day and night, in both constant darkness and under a 12:12 light-dark (LD) cycle. Using in vivo optogentically-targeted single unit activity recording, we demonstrated that these slow calcium waves are likely the result of burst-firing characteristic of AVP neurons previously reported for other brain regions. Rhythmicity analysis of several fluorescence measures suggests that individual AVP neurons exhibit unstable and stochastic rhythms, with approximately $30 \%$ of the neurons rhythmic during any given day across lighting conditions, and weak or absent rhythmicity at the population level. Network-level cross-correlational analysis revealed that coherence among neuron pairs also exhibited stochastic rhythms with about $25 \%$ of pairs rhythmic at any time. Notably, this analysis revealed a stronger rhythm at the population level than was observed in single cell analysis. The peak time of maximal coherence among AVP neuronal pairs occurs between CT/ZT 6 and 9, coinciding with the timing of maximal neuronal activity with the SCN as a whole. These results are the first to demonstrate robust circadian variation in the coordination between apparently weakly rhythmic or arrhythmic neurons suggesting that, for AVP neurons, interactions between neurons in the SCN are more influential than individual or single subpopulation activity in the regulation of mammalian circadian rhythms.
\end{abstract}




\section{Introduction}

In mammals, circadian rhythms are synchronized by the suprachiasmatic nucleus (SCN) of the ventral hypothalamus[1, 2]. At the molecular level, circadian rhythms are driven by a transcriptional/translational feedback loop in which the core clock genes CLOCK and BMAL1 promote the transcription of the Cryptochrome and Period genes, which in turn inhibit their own transcription in a process that takes approximately 24 hours[3]. While most mammalian cells contain this molecular machinery[4], with few notable exceptions $[5,6]$, rhythmicity is lost after only a few cycles when isolated from the SCN[7]. SCN neurons, cultured in isolation or experimentally separated, exhibit weak or unstable rhythms [4, 8-10]. Thus, although molecular clock machinery is necessary for the generation of circadian rhythms, individual neurons are insufficient to maintain longitudinal rhythms. There is increasing evidence that the neurons in the SCN act as a neural network in which interactions between neurons within the circuit may be responsible for regulation circadian rhythms. SCN brain slices exhibit robust rhythms in core clock gene expression and intracellular Calcium dynamics ex vivo for weeks to months[7, 11], and these rhythms are reversibly dampened when cellular communication is disrupted with TTX[11-13]. In addition, longitudinal bioluminescent slice recordings reveal the existence of an organized "wave" of clock gene expression which propagates through the SCN in a stereotypic fashion, for both mPer1[14] and $m$ Per2[11, 15]. This temporal organization is modified in a predictable and reversible way by alterations in daylength, suggesting that this emergent property of the network encodes important information about the external world [16, 17]. It remains unknown which SCN neurons are responsible for creating this coordinated activity.

While most SCN neurons are GABAergic[18], the SCN can be somewhat divided into a ventral "core" compartment containing vasoactive intestinal polypeptide (VIP)- and gastrin releasing peptide (GRP)-expressing GABAergic neurons, and a dorsal "shell" containing GABAergic neurons expressing arginine vasopressin (AVP)[19]. In addition, approximately $40 \%$ of SCN neurons express the neuropeptide Neuromedin S (NMS) and approximately $60 \%$ express the dopamine receptor DRD1[20]; however, these genes are not expressed in distinct neuronal populations and these neurons may also express AVP or VIP[21]. Activity of these subpopulations may correspond to differential roles within the circadian circuit. For instance, loss of NMS neurons abolishes rhythmicity entirely[21]. VIP- and GRP-expressing neurons likely receive input from the retina and play a role in entrainment of the SCN to the light/dark cycle[22, 23]. Also, VIP and GABA act together to maintain synchrony between SCN neurons, particularly under conditions of changing environmental lighting[16]. However, the role of AVP neurons is less clear. Disrupting the circadian clock by selectively knocking out either bmal1 or case kinase 1 delta in AVP neurons lengthened the period of oscillation[24-26], although selective destruction of AVP neurons themselves does not affect locomotor activity rhythms[27]. Taken together, available data suggest that rhythmicity of the SCN network is rather resistant to the loss of specific neuronal populations of the SCN network. The organization of this network is still poorly understood, but the ability to study the intact network in vivo using novel imaging and recording approaches presents an opportunity to answer this critical question in chronobiology. 
Multi-unit neuronal activity (MUA) recordings show that the intact SCN network exhibits a robust firing rhythm peaking during the subjective day both in DD and LD [28-31]. Intersectional approaches allow the examination of cell-type specific contributions to circadian timing in the intact SCN. Observation of VIP neuronal gene expression and intracellular calcium via fiber photometry of fluorescence show robust population level rhythms in calcium, Per1, Per2, and Cry1 in vivo[23, 32]. This approach highlights the importance of VIP neurons for photic entrainment[23, 33], and the importance of GABA release from AVP neurons in regulating the timing of firing of other SCN neurons[34]. However, fiber photometry is a population measure that does not provide the cellular resolution needed to enumerate how neural networks are formed from individual neurons. In the present work, we characterize bursting electrical behavior of AVP neurons within the intact SCN network using optogenetically-targeted single-unit activity recording (OSAR). Further, we employ miniaturized fluorescence microscopy to characterize a portion of the SCN network by conducting longitudinal recording of intracellular Calcium dynamics in AVP-expressing neurons in the fully intact SCN of freely behaving mice. We report, somewhat surprisingly, that individual AVP neurons appear to be relatively unstable circadian oscillators in vivo, exhibiting a stochastic pattern of rhythmicity in calcium dynamics over time. Interestingly however, correlational network analysis reveals a strong, stable circadian pattern of coherence among AVP neuron pairs at the population level, peaking during the circadian daytime. This study is the first to undertake cell-type-specific in vivo recordings of individual SCN neurons and demonstrate that AVP neuronal rhythms emerge largely at the network level.

\section{Methods}

\section{Animals}

For in vivo calcium imaging experiments, 10-week-old heterozygous male AVP-IRES2Cre-D (Jackson Laboratory 023530) were used. Control experiments were conducted using male Ai6 mice which express robust ZsGreen1 fluorescence following Cremediated recombination (Jackson Labs 007906) crossed with AVP-IRES2-CRE-D knock-in mice (Jackson Labs 023530). For in vivo optogenetically targeted single-unit recording, Mice were bred by crossing AVP-Cre with Ai32 (RCLChR2(H134R)/EYFP,Stock \# 024109) mice. In vivo electrophysiological recordings were performed on adult male mice (3-4 months) housed in 12L:12D light-dark cycle from birth. Food and water were available ad libitum. All procedures described were approved by the IACUC committee at Morehouse School of Medicine.

\section{In vivo Miniaturized Fluorescent Calcium Imaging}

Chronic lens implantation surgeries were done as previously described[35]. Briefly, using a stereotaxic apparatus, AVP-cre mice were injected (ML: +0.750, AP: -0.200 , DV: $-5.8 \mathrm{~mm}$ ) with an adeno-associated viral vector conveying the genetically encoded calcium sensor jGCaMP7s (\#104463, Addgene) into the SCN and a gradient index lens $0.50 \mathrm{~mm}$ in diameter (1050-004611 Inscopix) was implanted above the SCN (ML: +0.750 , AP: -0.200 , DV: $-5.7 \mathrm{~mm}$; Figure 1A). Six weeks later, mice were assessed for 
evidence of discrete, dynamic calcium signals and a baseplate was attached over the gradient index lens to establish a fixed working distance with the microscope (Figure 1B). Animals were allowed two days to acclimate to recording chamber and the presence of the microscope, then were subjected to three weeks of experimental recording (Figure 1C; $\mathbf{n}=\mathbf{3}$ mice; 33 AVP neurons). The recording schedule consisted of 5-minute recordings where samples were imaged at $6.67 \mathrm{~Hz}$, every three hours for 48 consecutive hours for a total of 16 timepoints under both constant darkness and 12:12LD (Figure 1D). This schedule was used to minimize the occurrence of photobleaching from over-excitation of GCaMP in the neurons. After completion of the recording schedule, mice were sacrificed and lens placement over the SCN was validated by histology (Figure 1E) and specificity of GCaMP infection was verified by colocalization IHC (Figure 1F). Colocalization was difficult to detect, as expected due to the reduced expression of the AVP peptide in AVP-cre mice[36]. GCaMP7s never colocalized with neurons expressing VIP, supporting AVP-expressing neuronal specificity. Locomotor activity was measured to verify that implanted mice had unimpaired circadian rhythms (Figure 1G). Proper functionality of the calcium sensor was established by comparing dynamic GCaMP7s recordings with GFP expressing AVP neurons used as a control (Figure 1H). The GFP signal appeared to be stochastic noise in all recordings, never exhibiting dynamics like those observed using the calcium indicator.

\section{In vivo Optogenetically-targeted Single-unit Activity Recording}

\section{Optrode Implantation}

Optrode Implantation was done as previously described[37]. Briefly, a stereotaxic apparatus (KOPF, model 1900) was used for implanting custom 12-channel optrodes above the SCN (AP: 0.38mm, ML: 0.15mm, DV: $-5.5 \mathrm{~mm}$ ). Optrodes were drivable via manual screw-drive, and once implanted were slowly advanced into the SCN over days/weeks (see below). Optrodes were constructed by combining a microelectrode

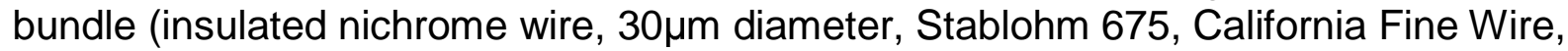
USA) with a custom-made optical fiber (200 $\mu \mathrm{m}$ diameter, Doric Lenses). The entire site was then sealed and secured with dental acrylic.

$\underline{\text { Identification of ChR2-Expressing Neurons and Data Acquisition }}$

After recovery from electrode implantation surgery, the mice were handled to adapt to extracellular recording procedures (30 $\mathrm{min})$ and the optrode is gradually lowered through the brain toward the SCN $(40 \mu \mathrm{m}$, once a day) until light stimulation through the optrode yielded single unit responses. For optogenetic identification of ChR2-expressing (AVP+) neurons, pulses of blue light (473 nm, 2-10 ms duration, $20 \mathrm{~Hz}, 10-15 \mathrm{~mW}$ ) were delivered to the SCN through the optical fiber. A single unit was identified as a ChR2-expressing neuron when action potential spikes were repeatedly and reliably evoked by the blue light pulses ( $>50 \%$ occurrence) with a short first-spike latency $(<7$ $\mathrm{ms}$ ), low jitter (<3 ms) and with waveforms consistent with prior-recorded spontaneous spikes (correlation coefficient between waveforms of spontaneous and optically evoked 
spikes $>97 \%$ ). Electrophysiological data were recorded with a sampling rate of $20 \mathrm{KHz}$ by a 16-channel head-stage and controller (RHD2000, Intan Technologies). The spontaneous firing of both laser-responsive (ChR2 positive) and non-responsive neurons were recorded continuously for 48 hours under the existing 12:12 light - dark cycle and followed by 48 hours under constant dark. Recorded raw voltage data was high-pass filtered (>250 Hz) and spikes were identified by applying thresholding. Single units were identified through offline sorting and then analyzed using NeuroExplorer and Matlab software. All electrode locations were verified histologically.

\section{Image Analysis and Statistics}

\section{Image Correction, ROI Definition and Production of Intensity Traces}

Raw image stacks acquired at $6.67 \mathrm{~Hz}$ were motion-corrected using Inscopix Data Processing software and five-minute recordings of images were exported as TIFF stacks. The TIFF stacks were imported into Igor Pro 8 (Wavemetrics Inc, Lake Oswego, Oregon) for further analysis. For each TIFF stack, a maximum projection image was then produced by finding the maximum intensity for each pixel during the 5-minute recording. This maximum projection image was used to identify regions of interest (ROIs) and regions of background. For a particular animal, ROI locations could vary slightly over the $24-48$ hours of experimentation, and thus, ROI locations could be slightly corrected from 3-hour time point to time point. Background was subtracted from each image in the stack by doing a two-dimensional interpolation of background intensities based on the background regions outlined in the maximum projection image. Using the background subtracted image stack, the average intensity for each ROI was determined for each image in the stack. This produced a line trace of varying intensities with time over the five-minute period for each ROI (e.g., Figure 2A).

\section{Mean Intensity, Intensity Correlation Analysis and Events Analysis}

One main focus of this study was to characterize if activity of individual neurons varied with circadian rhythmicity. Thus, we characterized each ROI for its average intensity within the five-minute trace at each 3-hour time point. Fluorescence intensity traces for each ROI were also cross-correlated with each other yielding a Pearson Coefficient for each ROI-ROI interaction at each circadian time point. We wished to determine if the power in the cross-correlations mainly resulted from slow frequencies below $1 \mathrm{~Hz}$ or faster frequencies. To do this, we subjected raw data to high or low pass digital Finite Impulse Response filters before cross-correlation analysis. Parameters for the low pass filter were: End Band Pass: $0.25 \mathrm{~Hz}$; Start of Stop Band: $0.5 \mathrm{~Hz}$; Number of Computed Terms: 73. Parameters of high pass filtering were: End of First Band: $0.5 \mathrm{~Hz}$; Start of Second Band: $1 \mathrm{~Hz}$; Number of terms: 41 . Edge effects were removed by deleting the first 17 and last 17 points in the filtered data trace. After cross-correlation analysis, the resulting Pearson Coefficients were analyzed by determining the weights that each of correlation of the filtered traces would contribute to the correlation coefficient that was not digitally filtered:

$$
P_{\text {unfiltered }}=w_{\text {low pass }} P_{\text {low pass }}+w_{\text {high pass }} P_{\text {high pass }}
$$

where $P$ represents the Pearson Coefficient determined for unfiltered data or for the Pearson Coefficient after filtering the data with either high or low pass filters. $w$ 
represents the contributing weights of each Pearson Coefficient to the Pearson Coefficient determined for the unfiltered data. An additional constraint was that

$$
W_{\text {low }} \text { pass }+W_{\text {high pass }}=1
$$

In addition, upon import of fluorescent images generated from the AVP neurons, we noticed slow fluctuations in fluorescent intensity $(\sim 10-30 \mathrm{~s})$ within our five-minute recordings in many ROls (Figures 1B, 4A). Thus, event parameters would need to be characterized. This was accomplished in two different ways. An unbiased analysis of events (Figure 3A) was conducted in which the onset of an event was found if the amplitude increased by more than two standard deviations above the intensity of the previous 20 points (3 seconds). Data was smoothed prior to this analysis using a 30point box window. A second method was used to determine event parameters of slow calcium waves. In a first pass, automatic analysis was done in which the raw fluorescence line trace for each ROI was smoothed with a 50-point box window. Then, the first derivative was calculated from the smoothed raw data trace. The first derivative trace was also smoothed using a 100-point box window followed by recording the time when a threshold level on the first derivative was crossed in an increasing manner when searching from the start of the first derivative trace to the end for either positive or negative slopes. This approximated the start of slow calcium fluorescence wave rise and the end of the calcium wave. The value of the threshold of the first derivative was determined heuristically (usually 0.05 ). These time points could then be manually corrected for missing and/or spurious events. Wave durations, inter-event intervals and wave number could then be calculated from the start and end times of each event in each fluorescent intensity line trace.

\section{Circadian Rhythm Analysis}

Circadian Rhythmicity was tested by fitting data collected at three-hour intervals over 24 or 48 hours with a cosine function as follows:

$$
y=\frac{A}{2} \cos \left(\frac{2 \pi(t-\psi)}{\theta}\right)+\frac{A}{2}+A_{o}
$$

where $A$ represents the amplitude of the cosine signal, $A_{\circ}$ is the average offset of the fluorescence signal from zero, $t$ is the time of each data point in hours, $\psi$ and $\theta$ are the phase and period respectively in hours resulting from the fit. The data that were fit with Equation 2 to determine rhythmicity were the mean intensities, intensity correlations between ROls, the wave number for dynamic calcium events as well as the number of calcium waves. Before fitting mean intensities, data was subjected to linear detrending to compensate for any consistent, progressive loss of fluorescence intensity over the period of the experiment. Fit optimization was performed using the internal Igor Pro algorithm based on Levenberg-Marquardt least-squares method constraining the results for the period between 21 and 28 hours[38, 39]. $p$ values from these fits were determined using the Igor Pro implementation of the non-parametric Mann-Kendall tau test. A measure for a particular ROI was deemed rhythmic if the $p$ value was below 0.05 . The 24 - or 48 -hour data was then ranked by $p$ value and displayed as heatmaps. Data which could not be fit with Equation 2 was given a $p$ value of 1 in the resulting heatmaps and is not ranked in any particular order. 
Phases $(\psi)$ and periods $(\theta)$ resulting from the fits to Equation 2 were saved for each $\mathrm{ROI}$ or ROI-ROI interaction with significant rhythmicity. Histograms of the phases were then calculated utilizing 3-hour bins and then plotted on polar coordinate graphs.

To determine if AVP neurons displayed any rhythmicity as a population, the columns of the heatmaps were averaged, and these values subject to the circadian fit using Equation 2. For population averages, fitting was also weighted by the reciprocal of the standard deviations determined for each column of the heatmap.

\section{Calculation of Firing Rate and Duration of Bursting for Single Unit Analysis}

After spike sorting, traces dedicated to a particular single unit were analyzed for their firing rate. Time stamps of action potential firing were subjected to histogram analysis with a bin size of $3 \mathrm{~s}$ for over the whole recording and then divided by the bin size to get the firing rate per $3 \mathrm{~s}$ time point. A histogram of this firing rate time course was then done, yielding a histogram indicating the prevalence of firing rates. Burst lengths were determined by rebinning the time stamp data per $10 \mathrm{~s}$ bins and determining the duration of the burst by analyzing the points when the firing rate had risen above and then returned below $1.5 \mathrm{~Hz}$. $1.5 \mathrm{~Hz}$ was determined empirically, as it seemed to correlate with bursting behavior observed by eye. Because firing rates and burst lengths could have a range of several decades, these histograms were converted to a log transform where the abscissa was the log of the firing rate and the ordinate was the square root of the number of events per bin (e.g. Figures $6 \mathrm{G}, 6 \mathrm{H}$ )[40]. This data was then fit with a log transformation of a sum of exponential components using the following equation[41]:

$$
y=A_{0}+\sum_{i}^{n}\left(\frac{A_{i}}{\iota_{i}} e^{\left(t-\frac{e^{t}}{\iota_{i}}\right)}\right)
$$

where $A_{\mathrm{i}}$ is the amplitude of the exponential of component $i, A_{0}$ is the amplitude offset, $l_{\mathrm{i}}$ is the exponential time constant of component $i$ and $n$ is the number of exponentials in the log transform. $t$ is the firing rate or the burst length for Figures $6 \mathrm{G}$ and $6 \mathrm{H}$, respectively.

\section{$\underline{\text { Results }}$}

\section{Circadian Rhythmicity in Mean Fluorescence of AVP Neurons in the SCN}

Circadian analysis of mean fluorescence intensities could indicate if basal levels of calcium, or overall levels of neuronal activity would change over the course of the day. Average fluorescence intensity was determined from each 5-minute recording at each three-hour time point for each ROI (Figure 2A). The mean fluorescence per three-hour time point was fit with equation 2 for each ROI to determine if the ROI exhibited a circadian rhythm. In constant darkness, 24\% of AVP neurons exhibited significant circadian rhythmicity with mean peak phase at CT $10.46 \pm 1.49$ hours; $n=6$ (Figure 2B above the arrow; Figure 2C); whereas in 12:12 LD, 53\% of the AVP neurons demonstrated significant circadian rhythmicity with a mean peak phase at ZT $9.11 \pm$ 1.80 hours; $n=18$ (Figure 2B above the arrow; Figure 2C). 
For each individual 24-hour day, approximately 35\% of recorded cells were rhythmic (Figure 2D), but the set of cells that made up that rhythmic subpopulation was highly dynamic, with neurons gaining or losing rhythmicity in the two 24-hour periods (Figure 2E left). Also, there was no discernable pattern between lighting conditions except for an overall increase in the likelihood of rhythmicity in LD compared with DD (Figure 2E right). When mean fluorescence values for all AVP neurons were averaged for each 3hour time point, the population did not display circadian rhythmicity in constant darkness (Figure 2B; $\mathbf{p}=\mathbf{0 . 5 4 6} ; \mathbf{n = 2 6}$ ). In contrast, under a 12:12 LD cycle the population average resulted in a significant population rhythm in mean fluorescence (Figure 2B; $\mathbf{p}$ $=0.005 ; n=34$ ).

\section{Rhythmicity of Dynamic Calcium Events in AVP Neurons}

Although slower variants of GCaMP including GCaMP7s used in this study are not capable discerning rapid events such as action potentials, significant changes in fluorescence within a recording are likely indicative of neuronal activity in these AVP neurons. Within the 5-minute recordings, events were detected automatically by finding fluorescence that crossed a threshold that was two standard deviations above the local background (Green Triangles; Figure 3A). In DD, 50\% of AVP neurons exhibited a circadian rhythm in dynamic calcium events compared with $44.1 \%$ of neurons in LD. The sub-population average phase for these dynamic events for these rhythmic cells in DD was at CT $14.21 \pm 1.30 ; n=13$ hours with a similar population average phase for LD at ZT $14.10 \pm 1.68$ hours; $n=15$ (Figure 3B above the triangle; Figure 3C). However, when considering all characterized AVP neurons (both rhythmic and non-rhythmic), the population average exhibited no significant rhythm in DD (Figure 3B; $\mathbf{p}=\mathbf{0 . 3 2 2 ;} \mathbf{n}=26$ ) or LD (Figure 3B; $\mathbf{p}=\mathbf{0 . 6 5 2} ; \mathbf{n = 3 4}$ ). As was observed for mean fluorescence (Figure 2D), there was a fairly consistent $\sim 30 \%$ of neurons that were rhythmic for any given 24 hour period (Figure 3D), but the members of that rhythmic subset (and sometimes the phase of those rhythms (not shown)) were highly dynamic across 48-hour and between lighting conditions (Figures 3E).

\section{AVP Neurons in the SCN Exhibit Distinctive Calcium Waves in vivo}

Slow, high amplitude calcium waves appear to be a characteristic feature of most AVP neurons in the SCN. The waves occurring within each 5-minute recording were counted (Figure 4A) across the circadian day to determine if the frequency of these events is rhythmic. In DD, calcium waves were observed in $87.7 \%$ of AVP neurons, though a significant circadian rhythm in their counts was observed in only $16.7 \%$ of AVP neurons with a peak phase at CT $14.24 \pm 0.96$ hours; $n=5$ (Figure 4B above the triangle, Figure 4C). Under 12:12 LD, calcium waves were observed in $76.5 \%$ of neurons, and significant circadian rhythms were detectable in $26.5 \%$ of the cells with a peak phase at ZT $20.06 \pm 1.79$ hours; $n=9$ (Figure 4B above the arrow, Figure 4C). Averaging the number of waves by timepoint for all AVP neurons did not reveal a significant circadian population rhythm in DD or in LD (Figure 4B; $p=1(n=26) ; p=0.133(n=34)$ respectively). Such waves were never observed in control experiments in which AVP neurons expressing GFP under the CAG promoter were recorded using the same methods, in either DD or LD (not shown). In addition to the analysis of wave number shown here, wave duration (Supplemental Figure S1) and inter-event interval between 
these calcium waves (Supplemental Figure S2) were also calculated but neither provided any evidence of stable circadian rhythmicity. As reported above for both mean fluorescence and calcium events, very little rhythmic stability for the number of calcium waves was observed over 2 days for individual neurons while $15-30 \%$ of neurons were rhythmic for 24-hour periods (Figure 4D). For the changing light conditions, largely different populations of cells were rhythmic in DD or LD (Figure 4E).

\section{Calcium Waves Correspond to Bursting Activity in AVP Neurons}

Optogenetically-targeted Single Unit Activity Recording (OSAR) was employed to determine if the timing of the slow wave phenomenon observed in calcium imaging (Figure 4) corresponded to increased action potential firing activity in individual AVP neurons within the intact SCN (Figures 5A, 5B). Mice expressing channelrhodopsin in AVP neurons were implanted with optrodes and neurons were considered to be AVP positive if an increase in firing rate was elicited by blue light stimulation through the optrode (Figure 5C). The latency to action potential firing after light stimulation was 2.26 $\mathrm{ms} \pm 0.02 \mathrm{~ms}(\mathrm{n}=100$ randomly selected APs; Figure 5D). When the firing rate was considered for 5-minute epochs, a distinct pattern of bursting was observed (Figure 5E) which corresponded closely with the calcium imaging data observed from SCN AVP neurons in other animals (Figure 5F). Both firing rate (Figure 5G) and burst duration (Figure $5 \mathrm{H}$ ) were calculated using the unbiased method described above. Average burst length was $13.41 \pm 2.6$ seconds and $8.92 \pm 2.11$ seconds in $D D(n=3)$ and LD $(n=4)$ respectively compared with the calcium wave duration of $14.20 \pm 0.50$ seconds and $13.97 \pm 0.32$ seconds in DD and LD respectively (Figure 5I). Two-way ANOVA indicated that there was no main effect of method of observation ( $D F=1, F_{1,677}=1.27$, $p=0.26 ; n=7$ ) or lighting condition ( $\left.D F=1, F_{1,677}=0.827, p=0.37, n=7\right)$ as well as no interaction $\left(D F=1, F_{1,677}=0.67, p=04.1, n=7\right.$ ).

\section{Rhythmicity of Correlated Activity between AVP Neurons}

Raw fluorescence across all cell pairs within a recording (Figure 6A) was subjected to correlational analysis for each time-point to determine the degree of coherence of intracellular calcium changes among AVP neurons, and to determine if the degree of correlation varies over circadian/diurnal time. We observed that many pairs exhibited strong positive correlations during the middle of the subjective day and either no correlation or were inversely correlated during the subjective night (Figure 6A, Right). In constant darkness, $30.23 \%$ of AVP neuron pairs exhibited a circadian rhythm in correlated activity in DD with an average peak correlation among rhythmic cells at a phase of CT $10.18 \pm 0.79$ hours; $n=38$ (Figure 6B above the triangle; Figure $6 \mathrm{C}$ ). In LD, $30.25 \%$ of AVP neurons demonstrated significant rhythmicity with a peak phase at ZT $8.11 \pm 0.59$ hours; $n=49$ (Figure 6B above the triangle; Figure $6 \mathrm{C}$ ). The population average of Pearson coefficients by timepoint exhibited a significant rhythm at the population level for all cells in both DD (Figure 6B; $p=0.038 ; n=160$ ) and LD (Figure $6 B ; p=0.037 ; n=188$ ).

We wanted to know how much of the power in the correlations resulted from low frequency fluorescence changes $(<0.25 \mathrm{~Hz})$ or if higher frequency calcium dynamics were mostly responsible for the interactions between AVP neurons. Frequency 
contribution analysis on a subset of interactions indicated that wave components slower than $0.25 \mathrm{~Hz}$ accounted for $99.2 \pm 0.40 \%$ of the correlation between AVP neurons $(\mathrm{n}=$ 10; Figure 6D), indicating that an increased synchronous activity was arising from coincidence in calcium waves or other slow dynamics in AVP neuronal pairs rather than due to shared high-frequency dynamics.

Rhythmicity in correlated activity consistently occurred in 25-35\% of AVP neuron pairs across all 4 of the 24-hour periods for which we recorded (Figure 6E), but the membership of that rhythmic subset of cell pairs was highly variable. Cell pairs either gained, lost, or maintained their rhythmic relationship across the 48-hour recordings (Figure 6F left) and between DD and LD (Figure 6F right) with no predictability. The strength of correlation as a function of distance between neurons was also measured. In $\mathrm{DD}$, there was a weak but significant relationship between correlated activity and the distance between AVP neurons, such that the further one neuron was from another for both positively $\left(R^{2}=0.007, p<0.001 ; n=160\right.$; not shown) and negatively correlated relationships $\left(R^{2}=0.01, p<0.004 ; n=160\right.$; not shown), Pearson Coefficients decreased. Correlated activity also weakly decreased with distance between neurons for 12:12LD, for both positively correlated $\left(R^{2}=0.02, p<0.001 ; n=188\right.$; not shown) and negatively correlated $\left(R^{2}=0.04, p<0.001 ; n=188 ;\right.$ not shown).

\section{$\underline{\text { Discussion }}$}

The master circadian pacemaker in mammals in the suprachiasmatic nucleus (SCN) is comprised of groups of neurons which express distinct neuropeptides and these cells may exert distinct influence over the regulation of circadian rhythms. It is still poorly understood how this network generates a unified output in which different neurons within the network interact to regulate circadian rhythmicity. Slice imaging, using bioluminescent and fluorescent gene-reporter mice, demonstrate the existence of robust network organization that is predictably and reversibly altered by changing light conditions[11-13, 42]. However, this approach is limited by the need to dissect the SCN network for observation, severing all inputs, outputs, and most intra-network connections. Multi-unit recording offers a window into the electrical activity of the intact SCN network in vivo, and such studies have been fundamental in developing our understanding of the SCN as a master pacemaker for rhythmicity [28-31, 43], but are unable to provide additional information regarding cell-type-specific population or individual neuronal contribution to the regulation of circadian rhythmicity within the SCN.

Intersectional genetics and novel recording techniques have dramatically improved our ability to conduct population level, cell-type specific studies within the intact circadian network. For example, recent studies in vivo demonstrate the necessity and sufficiency of VIP-expressing neurons in synchronizing the SCN to light-induced phase resetting[23, 32, 33]. In vivo fiber photometry is a powerful technique, but one that can be enhanced with the ability to observe individual neurons and the interactions between these neurons within the fully intact SCN network. Thus, here we present work involving two novel techniques: optogenetically-targeted single unit activity recording (OSAR) and in vivo calcium imaging of AVP-expressing neurons in the completely intact SCN of awake, freely moving mice under different lighting schedules using miniaturized fluorescence microscopy. OSAR has been used for cell-type specific 
electrophysiological recording in the medial prefrontal cortex[44], and the striatum[45], but applied here for the first time in the SCN. In vivo calcium imaging has been used to measure calcium dynamics in many brain areas including the frontal cortex[46], hippocampus[47], and cerebellum[48, 49]. This technique permits the characterization of individual nodes within the SCN and how they contribute to communal network behavior, and how these contributions are modulated over circadian/diurnal time.

Our data indicate that most AVP neurons in the SCN exhibit slow calcium 'waves', approximately $20 \%$ of which show a modulatory rhythm of their frequency over a 24 hour period (Figure 4). While increases in intracellular calcium can be indicative of neuronal firing [50], because of the slow decay time of the variant of GCaMP7 we employed, temporal discrimination of individual action potentials within bursting events was not possible. Thus, we employed OSAR to better characterize the firing behavior of AVP neurons within the SCN in vivo. The burst analysis resulting from firing rate of AVP neurons by OSAR is similar to the duration of calcium waves measured by fluorescence (Figure 5I). This suggests that the waves we observe in our calcium imaging data are likely the result of bursting activity exhibited by AVP neurons. Previous studies observed similar bursting activity in AVP neurons of the supraoptic nucleus[51] and paraventricular nucleus[52] .Although the specific cell-types were not identified, such bursting is also reported within the SCN[53]. The present results are the first to bridge these findings and confirm that AVP neurons within the SCN exhibit phasic firing, or bursting activity, in vivo. Interestingly, although bursting activity is present in nearly all SCN AVP-neurons recorded, a majority of neurons do not exhibit circadian rhythmicity in bursting. This suggests that while bursting is an identifying characteristic of these neurons, it does not play a significant role in the regulation of circadian rhythms.

Although circadian or diurnal regulation of bursting is uncommon in SCN AVP+ neurons (Figures 4, S1, S2), the well-known role of these cells as regulators of the circadian clock suggests that some aspect of their activity should exhibit reliable rhythmicity. AVPspecific Per2::Iuc rhythms in SCN slices illustrate the presence of functional molecular clocks[26]. Imaging from SCN slices from AVPEluc/+ mice demonstrates the rhythmic transcription of avp in the SCN[54], and there is a circadian rhythm in the secretion of vasopressin[55, 56] and GABA[34] from AVP neurons in the SCN. Using longitudinal calcium imaging, we find that stable circadian rhythmicity is not observed in all AVP neurons. Not even a majority of these neurons exhibited rhythmicity for the parameters characterized (mean fluorescent intensity, calcium events, or calcium waves). While individual neurons exhibit circadian rhythmicity in various measures, sometimes, these rhythms are not stable from day to day nor is there a reliable trend in rhythmicity between lighting conditions (Figures 2E, 3E, 4E, 6F). Furthermore, the phases of the calcium rhythms that we did observe vary among cells as well as across time/lighting condition such that rhythms do not emerge at the population level in most of these measures. Thus, while AVP neurons exhibit strong rhythms in gene transcription with coherent phasing in slice recordings, such rhythmicity may not accurately reflect the temporal activity of these neurons in vivo. This characteristic may be specific to AVP expressing neurons, since population level rhythms are observed in VIP neurons in vivo[23, 32, 33]. The apparently stochastic nature of rhythmic activity in AVP neurons presents a challenge given the importance of rhythmicity in AVP neurons. Loss of rhythmic GABA release from AVP neurons resulted in abnormal SCN firing and 
locomotor activity[34], and AVP-specific knockout of bmal1 results in mice that have significantly longer activity periods in constant darkness, decreased light

responsiveness, and that also rapidly re-entrained to 4-hour phase advances[24]. Taken together, these results suggest that while some aspect of rhythmicity in AVP neurons must be important for circadian regulation, perhaps stable rhythmic activity of individual neurons is not needed. Instead, perhaps a generous (and ever-changing) subset (i.e. $\sim 30 \%$ of cells) need only be rhythmic in order to drive rhythmic outputs. Our data suggest a novel view of network rhythmicity, where all cells contribute to the generation of a population rhythm, but not all the time.

The ability to endogenously generate and sustain stable circadian rhythmicity independent of environmental and extra-SCN circuit cues is a fundamental feature of the SCN. Recent work supports the hypothesis that emergent properties of the SCN network play an important role in creating this unique feature. The "wave" observed in bioluminescent slice recordings reveal a spatiotemporal organization within the SCN $[11,14,15]$ which is predictably and reversibly altered by changes in environmental lighting[16, 17]. Synchronization between cells may be dependent on this organization[57, 58] driven by region-specific circadian programming within the SCN network[42, 59, 60]. However, to date, it is unknown if such an organization observed ex vivo extends to an intact SCN network in vivo. Other approaches employed in vivo were not capable of single-cell discrimination and/or have not been able to observe multiple cells simultaneously, and therefore have not addressed the issue of network organization. It has been suggested that the SCN network is organized as a collection of weak oscillators which are driven by central pacemaker neurons ("small world" organization[61]). The role AVP neurons would play in such a schema is unclear since the overall rhythmicity of the network seems unable to reliably modulate calcium dynamics in a circadian manner. Neither do our observations yet support a "democratic" model of determining ensemble circadian characteristics. The general lack of rhythmicity in an important SCN population would seem to prevent that population from impacting rhythmicity in the broader population.

Interestingly, while individual AVP neurons in the SCN are weak, unstable circadian oscillators in vivo, there seems to be an underlying circadian structure to their coordinated activity. We observe that the slow calcium dynamics in many AVP cell pairs are more highly correlated during the day than during the night, indicating either coordinated function or a shared acute response to a common input (i.e., coherence) (Figure 6). This coherence in activity is dependent on distance. The further two neurons are from each other in the same field of view, the less correlated they are likely to be. In about $53 \%$ of the pairs, the relationship is absent during the night such that the cells behaved entirely independently, or sometimes are inversely correlated. Though only about $30 \%$ of AVP neuron pair relationships have significant circadian rhythms, the average of these correlations across all cell pairs, a surrogate population measure, in both DD and LD exhibit a significant circadian rhythm at the population level with a (subjective) daytime peak between CT/ZT 6-9. This in vivo observation of rhythms in coherence may reflect time-of-day dependent signaling dynamics in the SCN previously reported. GABA is known to switch between being inhibitory and excitatory in a time-ofday dependent manner [62-65], regulated via neuronal intracellular chloride concentration and expression of the $\mathrm{Na}^{+}-\mathrm{K}^{+}-2 \mathrm{Cl}^{-}$cotransporter (NKCC 1). The 
significance of this is demonstrated by time-dependent blockade of the NKCC1 transport, which results in diminished phase-resetting to light exposure during the early subjective night, but not during the late subjective night or during the light phase[66]. The relevance of this time-of-day dependent rhythm in neuronal coherence for circadian function needs further study.

The population measure of rhythmic coherence among AVP cell pairs further highlights the importance of emergent characteristics in the SCN neural circuit as well as the potential limitations of studying population level dynamics which ignore individual neuronal activity. While the use of cell-type specific AVP recording in this study helped to ensure we were observing network activity within the $\mathrm{SCN}$, the relative sparsity of this neuronal subtype made observing a large number of neurons prohibitive. Furthermore, conducting such analysis on only a single cell-type within a heterogenous network is inherently limiting. Therefore, future studies will focus on investigating the role of network organization in a more diverse, SCN-specific cell population. 
Figure Legends

Figure 1: in vivo Characterization of AVP Neurons in the SCN Network. A: Illustration describing AAV infection of AVP neurons and GRIN lens implantation above the SCN. B: Illustration and sample photomicrograph demonstrating how AVP calcium dynamics are observed in vivo. Green polygons are drawn around AVP neuronal regions of interest and the red polygons are non-AVP neuronal spaces used for background subtraction. Sample traces on the right illustrate examples of cell-traces obtained from AVP neurons in the same field of view. C: Timeline of in vivo calcium imaging experiments. D: Illustration describing the recording schedule: 5-minute recordings every three hours for 48 consecutive hours in both constant darkness and under 12:12 LD. E: Photomicrographs exhibiting histological sections which contain AVP neurons expressing GcaMP7s. White dashed rectangles represent the placement of the GRIN lens. 3V = third ventricle; OC = optic chiasm. F: Image of the SCN taken with a confocal microscope (63x lens) showing neuronal colocalization (yellow) of GcaMP7s (green) and AVP antibody (orange) as well as the exclusion of GcaMP7s from VIP expressing neurons (white). Red boxes indicate neurons with clear colocalization. G: Representative actogram demonstrating that mice with a GRIN lens implanted above the SCN appear to have normal daily circadian behavior which shifts normally in response to a 6-hour phase advance. H: Representative calcium traces recorded from the SCN of AVPcre x GFP mice and AVPcre mice infected with GcaMP7s demonstrating that while GFP does not exhibit acute calcium dynamics, biological release of intracellular calcium is detected by GcaMP7s.

Figure 2: Circadian Rhythmicity in Mean Fluorescence Intensity of Individual AVP Neurons. A: Representative 5-minute calcium trace demonstrating how mean fluorescence intensity (red dotted line) was used to measure calcium changes across the circadian day. B: Heat maps illustrating mean intensity of individual AVP neurons (rows) by timepoint (columns) in both DD and LD. Neurons which exhibited significant circadian rhythms in mean fluorescence appear above the dashed line and triangle. Plotted beneath are the population averages and standard errors for each timepoint and the p-value associated with population level circadian rhythmicity. C: Polar plots illustrating the phase distribution and percentage of AVP neurons exhibiting significant circadian rhythms in mean fluorescence in both DD and LD. D: Percent of recorded neurons that exhibited a statistically significant rhythm within each 24-hour recording day across the 48 hours of DD and LD. E: Phenotype tracking plots indicating the change in rhythmic state of individual AVP neurons between recording days under constant darkness (left) and between lighting conditions (right). Red lines indicate a loss of rhythmicity between conditions, green lines a gain of rhythmicity, and black lines indicate no change in rhythm state between conditions/recording days.

Figure 3: Circadian Rhythmicity in Dynamic Calcium Events of Individual AVP Neurons. A: Representative 5-minute calcium trace demonstrating unsupervised quantification of acute calcium events. B: Heat maps illustrating number of acute calcium events for individual AVP neurons (rows) by timepoint (columns) in both DD and LD. Neurons which exhibited significant circadian rhythms in the number of calcium 
events appear above the dashed line and triangle. Plotted beneath are the population averages and standard errors for each timepoint and the p-value associated with population level circadian rhythmicity. C: Polar plots illustrating the phase distribution and percentage of AVP neurons exhibiting significant circadian rhythms in the number of calcium events in both DD and LD. D: Percent of recorded neurons that exhibited a statistically significant rhythm within each 24-hour recording day across the 48 hours of DD and LD. E. Phenotype tracking plots indicating the change in rhythmic state of individual AVP neurons between recording days under constant darkness (left) and between lighting conditions (right). Red lines indicate a loss of rhythmicity between conditions, green lines a gain of rhythmicity, and black lines indicate no change in rhythm state between conditions.

Figure 4: Circadian Rhythmicity in Number of Calcium Waves of Individual AVP Neurons. A: Representative 5-minute calcium trace demonstrating quantification of calcium waves. Wave onset and end are indicated by the green and red triangles, respectively. B: Heat maps illustrating the mean number of calcium waves of individual AVP neurons (rows) by timepoint (columns) in both DD and LD. Neurons which exhibited significant circadian rhythms in the number of calcium waves appear above the dashed line with the arrow. Plotted beneath are the population averages and standard errors for each timepoint and the p-value associated with population level circadian rhythmicity. C: Polar plots illustrating the phase distribution and percentage of AVP neurons exhibiting significant circadian rhythms in the number of calcium waves in both DD and LD. A minority of neurons did not exhibit waves as indicated in the pie charts (black area). D: Percent of recorded neurons that exhibited a statistically significant rhythm within each 24-hour recording day across the 48 hours of DD and 48 hours of LD. E: Phenotype tracking plots indicating the change in rhythmic state of individual AVP neurons between recording days under constant darkness (left) and between lighting conditions (right). Red lines indicate a loss of rhythmicity between conditions, green lines a gain of rhythmicity, and black lines indicate no change in rhythmic state between conditions.

Figure 5: Electrical Characterization of Calcium Waves using OptogeneticallyTagged Single Unit Activity Recording in vivo. A: Schematic illustrating how laser stimulation is used to identify and record from AVP neurons in freely behaving mice. B: Photomicrograph of representative histological section illustrating the location of the fiber-optrode above the SCN. Blue=DAPI; Green=EYFP expression tag for Channelrhodopsin. $\mathrm{OC}=$ optic chiasm. C: Blue light stimulation yielded an increase in firing rate in AVP+ neurons containing channelrhodopsin. D: Histogram showing the the short latency between blue light stimulation and action potential firing in AVP neurons containing channelrhodopsin. E: Action potential firing measured from the optrode of a representative AVP neuron (top) with the smoothed firing rate plotted beneath. F: Representative 5-minute calcium trace of GCaMP mediated fluorescence from an AVP neuron. Note the similarity between the fluorescence calcium waves to the increased firing rate measured by OSAR plotted above. G: Example histogram of elevated firing rate duration for an AVP neuron. The black line indicates the fit to the log transform of the exponential fit according to equation 3 with the yellow lines being the individual 
exponential components from the fit. $\mathrm{H}$ : Example histogram from the same neuron showing the log transform of burst length. I: Summary of average burst duration recorded with OSRA (left) compared with the duration of calcium waves measured via miniaturized fluorescent microscopy (right). There was no significant difference in duration between recording modalities.

Figure 6: Circadian Rhythmicity in Coherence Among AVP Neuron Pairs. A: Schematic illustrating coherence analysis among AVP neuron pairs. Calcium traces of individual SCN neurons within a $5 \mathrm{~min}$ recording were cross-correlated, then the Pearson coefficient for cell pairs was plotted for each time point over 48 hours and subjected to rhythmicity analysis. Coherence among some pairs was strongly rhythmic. B: Heat maps illustrating Pearson's coefficients of single AVP neuronal pairs (rows) by timepoint (columns) in both DD and LD. Neuronal pairs which exhibited significant circadian rhythms in correlated activity appear above the dashed line and triangle. Plotted beneath are the population averages and standard errors for each timepoint and the p-value associated with population level circadian rhythmicity. C: Polar plots illustrating the phase distribution and percentage of AVP pairs exhibiting significant circadian rhythms in coherence in both DD and LD. D: Histogram illustrating the frequency contribution to neuronal pair correlation. A low-pass filter $(<0.25 \mathrm{~Hz})$ applied to raw data yielded correlations with Pearson Coefficients approximating the unfiltered data. On average, over $90 \%$ of the power was carried by these low frequencies in comparison to when a high-pass filter $(>1 \mathrm{~Hz})$ was applied to the raw data. E: Percent of recorded neurons that exhibited a statistically significant rhythm within each 24-hour recording day across the 48 hours of DD and 48 hours of LD. F: Phenotype tracking plots indicating the change in rhythmic state of AVP neuron pair coherence between recording days under constant darkness (left) and between lighting conditions (right). Red lines indicate a loss of rhythmicity between conditions, green lines a gain of rhythmicity, and black lines indicate no change in rhythm state between conditions.

\section{Supplemental Figure 1: Circadian Rhythmicity in Duration of Dynamic Calcium Events of Individual AVP Neurons. A: Representative 5-minute calcium trace demonstrating analysis of duration of calcium waves (time between the green (onset) and red (offset) triangles). B: Heat maps illustrating the duration of calcium waves of individual AVP neurons (rows) by timepoint (columns) in both DD and LD. Neurons which exhibited significant circadian rhythms in calcium wave duration appear above the dashed line and triangle. Plotted beneath are the population averages and standard errors for each timepoint and the $p$-value associated with population level circadian rhythmicity. C: Polar plots illustrating the phase distribution and percentage of AVP neurons exhibiting significant circadian rhythms in calcium waves duration in both DD and LD. D: Percent of recorded neurons that exhibited a statistically significant rhythm within each 24-hour recording day across the 48 hours of DD and 48 hours of LD. E: Phenotype tracking plots indicating the change in rhythm state of individual AVP neurons between recording days under constant darkness (left) and between lighting conditions (right). Red lines indicate a loss of rhythmicity between conditions, green lines a gain of rhythmicity, and black lines indicate no change in rhythm state between conditions.}


Supplemental Figure 2: Circadian Rhythmicity in Inter-event Interval of Dynamic Calcium Events of Individual AVP Neurons. A: Representative 5-minute calcium trace demonstrating analysis of calcium wave inter-event interval (time between the red (offset) triangle of one wave and the green (onset) triangles of the next wave). B: Heat maps illustrating the inter-event interval of calcium waves of individual AVP neurons (rows) by timepoint (columns) in both DD and LD. Neurons which exhibited significant circadian rhythms in inter-event interval of calcium waves appear above the dashed line and triangle. Plotted beneath are the population averages and standard errors for each timepoint and the $p$-value associated with population level circadian rhythmicity. C: Polar plots illustrating the phase distribution and percentage of AVP neurons exhibiting significant circadian rhythms in inter-event interval of calcium waves in both DD and LD. D: Percent of recorded neurons that exhibited a statistically significant rhythm within each 24-hour recording day across the 48 hours of DD and 48 hours of LD. E: Phenotype tracking plots indicating the change in rhythm state of individual AVP neurons between recording days under constant darkness (left) and between lighting conditions (right). Red lines indicate a loss of rhythmicity between conditions, green lines a gain of rhythmicity, and black lines indicate no change in rhythm state between conditions.

Acknowledgements: This work was supported by National Institute of Health grants R21NS108197 and R35GM136661 to AJD, SC1AG046907 to MB, and SC1GM127260 to JCE, and an equipment grant to MSM by the W.M. Keck Foundation. We also thank Ivory Ellis and Inscopix, Inc., for technical assistance with the project. 


\section{Cited References}

1. Ralph, M.R., et al., Transplanted suprachiasmatic nucleus determines circadian period. Science, 1990. 247(4945): p. 975-8.

2. Weaver, D.R., The suprachiasmatic nucleus: a 25-year retrospective. J Biol Rhythms, 1998. 13(2): p. 100-12.

3. Takahashi, J.S., Transcriptional architecture of the mammalian circadian clock. Nat Rev Genet, 2017. 18(3): p. 164-179.

4. Welsh, D.K., et al., Individual neurons dissociated from rat suprachiasmatic nucleus express independently phased circadian firing rhythms. Neuron, 1995. 14(4): p. 697-706.

5. Su Terman, J., C.E. Reme', and M. Terman, Rod outer segment disk shedding in rats with lesions of the suprachiasmatic nucleus. Brain Research, 1993. 605(2): p. 256-264.

6. Koronowski, K.B., et al., Defining the Independence of the Liver Circadian Clock. Cell, 2019. 177(6): p. 1448-1462.e14.

7. Yoo, S.-H., et al., PERIOD2::LUCIFERASE real-time reporting of circadian dynamics reveals persistent circadian oscillations in mouse peripheral tissues. Proceedings of the National Academy of Sciences of the United States of America, 2004. 101(15): p. 5339-5346.

8. Herzog, E.D., et al., Circadian rhythms in mouse suprachiasmatic nucleus explants on multimicroelectrode plates. Brain Research, 1997. 757(2): p. 285-290.

9. Honma, S., et al., Circadian periods of single suprachiasmatic neurons in rats. Neuroscience Letters, 1998. 250(3): p. 157-160.

10. Herzog, E.D., J.S. Takahashi, and G.D. Block, Clock controls circadian period in isolated suprachiasmatic nucleus neurons. Nature Neuroscience, 1998. 1(8): p. 708-713.

11. Noguchi, T., et al., Calcium Circadian Rhythmicity in the Suprachiasmatic Nucleus: Cell Autonomy and Network Modulation. eNeuro, 2017. 4(4).

12. Schmal, C., E.D. Herzog, and H. Herzel, Measuring Relative Coupling Strength in Circadian Systems. Journal of Biological Rhythms, 2018. 33(1): p. 84-98.

13. Yamaguchi, S., et al., Synchronization of Cellular Clocks in the Suprachiasmatic Nucleus. Science, 2003. 302(5649): p. 1408.

14. Hastings, M.H., et al., Differential regulation of $m P E R 1$ and $m T I M$ proteins in the mouse suprachiasmatic nuclei: new insights into a core clock mechanism. J Neurosci, 1999. 19(12): p. Rc11.

15. Enoki, R., et al., Topological specificity and hierarchical network of the circadian calcium rhythm in the suprachiasmatic nucleus. Proceedings of the National Academy of Sciences, 2012. 109(52): p. 21498.

16. Evans, J.A., et al., Dynamic interactions mediated by nonredundant signaling mechanisms couple circadian clock neurons. Neuron, 2013. 80(4): p. 973-83.

17. Rohr, K.E., et al., Seasonal plasticity in $G A B A(A)$ signaling is necessary for restoring phase synchrony in the master circadian clock network. Elife, 2019. 8.

18. Moore, R.Y. and J.C. Speh, GABA is the principal neurotransmitter of the circadian system. Neurosci Lett, 1993. 150(1): p. 112-6.

19. Abrahamson, E.E. and R.Y. Moore, Suprachiasmatic nucleus in the mouse: retinal innervation, intrinsic organization and efferent projections. Brain Res, 2001. 916(1-2): p. 172-91. 
20. Meng, Q.J., et al., Setting clock speed in mammals: the CK1 epsilon tau mutation in mice accelerates circadian pacemakers by selectively destabilizing PERIOD proteins. Neuron, 2008. 58(1): p. 78-88.

21. Lee, I.T., et al., Neuromedin s-producing neurons act as essential pacemakers in the suprachiasmatic nucleus to couple clock neurons and dictate circadian rhythms. Neuron, 2015. 85(5): p. 1086-102.

22. Park, J., et al., Single-Cell Transcriptional Analysis Reveals Novel Neuronal Phenotypes and Interaction Networks Involved in the Central Circadian Clock. Front Neurosci, 2016. 10: p. 481.

23. Jones, J.R., et al., SCN VIP Neurons Are Essential for Normal Light-Mediated Resetting of the Circadian System. The Journal of Neuroscience, 2018. 38(37): p. 7986.

24. Mieda, M., et al., Cellular Clocks in AVP Neurons of the SCN Are Critical for Interneuronal Coupling Regulating Circadian Behavior Rhythm. Neuron, 2015. 85(5): p. 1103-1116.

25. Mieda, M., H. Okamoto, and T. Sakurai, Manipulating the Cellular Circadian Period of Arginine Vasopressin Neurons Alters the Behavioral Circadian Period. Current Biology, 2016. 26(18): p. 2535-2542.

26. Shan, Y., et al., Dual-Color Single-Cell Imaging of the Suprachiasmatic Nucleus Reveals a Circadian Role in Network Synchrony. Neuron, 2020. 108(1): p. 164-179.e7.

27. Todd, W.D., et al., Suprachiasmatic VIP neurons are required for normal circadian rhythmicity and comprised of molecularly distinct subpopulations. Nature Communications, 2020.11(1): p. 4410.

28. Inouye, S.T. and H. Kawamura, Persistence of circadian rhythmicity in a mammalian hypothalamic "island" containing the suprachiasmatic nucleus. Proc Natl Acad Sci U S A, 1979. 76(11): p. 5962-6.

29. Yamazaki, S., et al., Rhythmic Properties of the Hamster Suprachiasmatic Nucleus\&It;em\&gt; In Vivo\&It;/em\&gt. The Journal of Neuroscience, 1998. 18(24): p. 10709.

30. Nakamura, W., et al., In vivo monitoring of circadian timing in freely moving mice. Curr Biol, 2008. 18(5): p. 381-5.

31. Takasu, N.N., et al., In vivo monitoring of multi-unit neural activity in the suprachiasmatic nucleus reveals robust circadian rhythms in Period1 ${ }^{-}$mice. PLoS One, 2013. 8(5): p. e64333.

32. Ono, D., K. Honma, and S. Honma, Circadian and ultradian rhythms of clock gene expression in the suprachiasmatic nucleus of freely moving mice. Sci Rep, 2015. 5: p. 12310.

33. Mazuski, C., et al., Entrainment of Circadian Rhythms Depends on Firing Rates and Neuropeptide Release of VIP SCN Neurons. Neuron, 2018. 99(3): p. 555-563.e5.

34. Maejima, T., et al., GABA from vasopressin neurons regulates the time at which suprachiasmatic nucleus molecular clocks enable circadian behavior. Proceedings of the National Academy of Sciences, 2021. 118(6): p. e2010168118.

35. Resendez, S.L., et al., Visualization of cortical, subcortical and deep brain neural circuit dynamics during naturalistic mammalian behavior with head-mounted microscopes and chronically implanted lenses. Nat Protoc, 2016. 11(3): p. 566-97. 
36. Cheng, A.H., S.W. Fung, and H.M. Cheng, Limitations of the Avp-IRES2-Cre (JAX\#023530) and Vip-IRES-Cre (JAX \#010908) Models for Chronobiological Investigations. J Biol Rhythms, 2019. 34(6): p. 634-644.

37. Cardin, J.A., et al., Targeted optogenetic stimulation and recording of neurons in vivo using cell-type-specific expression of Channelrhodopsin-2. Nature Protocols, 2010. 5(2): p. 247-254.

38. PES volume 2 issue 1 Cover and Back matter. Probability in the Engineering and Informational Sciences, 1988. 2(1): p. b1-b3.

39. Shrager, R.I., Quadratic programming for nonlinear regression. Commun. ACM, 1972. 15(1): p. 41-45.

40. Sigworth, F. and S. Sine, Data transformations for improved display and fitting of singlechannel dwell time histograms. Biophysical journal, 1987. 52(6): p. 1047-1054.

41. Colquhoun, D. and F. Sigworth, Fitting and statistical analysis of single-channel records, in Single-channel recording. 1995, Springer. p. 483-587.

42. Enoki, R., et al., Dual origins of the intracellular circadian calcium rhythm in the suprachiasmatic nucleus. Scientific Reports, 2017. 7(1): p. 41733.

43. Michel, S., et al., Electrophysiological Approaches to Studying the Suprachiasmatic Nucleus. Methods Mol Biol, 2021. 2130: p. 303-324.

44. Anikeeva, P., et al., Optetrode: a multichannel readout for optogenetic control in freely moving mice. Nature Neuroscience, 2012. 15(1): p. 163-170.

45. Kravitz, A.V., S.F. Owen, and A.C. Kreitzer, Optogenetic identification of striatal projection neuron subtypes during in vivo recordings. Brain Research, 2013. 1511: p. 2132.

46. Prevedel, R., et al., Fast volumetric calcium imaging across multiple cortical layers using sculpted light. Nature Methods, 2016. 13(12): p. 1021-1028.

47. Indersmitten, T., et al., Utilizing Miniature Fluorescence Microscopy to Image Hippocampal Place Cell Ensemble Function in Thy1.GCaMP6f Transgenic Mice. Current Protocols in Pharmacology, 2018. 82(1): p. e42.

48. Sullivan, M.R., et al., In Vivo Calcium Imaging of Circuit Activity in Cerebellar Cortex. Journal of Neurophysiology, 2005. 94(2): p. 1636-1644.

49. Díez-García, J., W. Akemann, and T. Knöpfel, In vivo calcium imaging from genetically specified target cells in mouse cerebellum. Neurolmage, 2007. 34(3): p. 859-869.

50. Fleming, W., et al., Inferring spikes from calcium imaging in dopamine neurons. PLOS ONE, 2021. 16(6): p. e0252345.

51. Gouzènes, L., et al., Vasopressin Regularizes the Phasic Firing Pattern of Rat Hypothalamic Magnocellular Vasopressin Neurons. The Journal of Neuroscience, 1998. 18(5): p. 1879.

52. Dewald, M., et al., Phasic bursting activity of paraventricular neurons is modulated by temperature and angiotensin II. Journal of Thermal Biology, 1999. 24(5): p. 339-345.

53. Miller, J.D. and C.A. Fuller, Isoperiodic neuronal activity in suprachiasmatic nucleus of the rat. American Journal of Physiology-Regulatory, Integrative and Comparative Physiology, 1992. 263(1): p. R51-R58. 
54. Yoshikawa, T., et al., Spatiotemporal profiles of arginine vasopressin transcription in cultured suprachiasmatic nucleus. European Journal of Neuroscience, 2015. 42(9): p. 2678-2689.

55. Södersten, P., et al., A daily rhythm in behavioral vasopressin sensitivity and brain vasopressin concentrations. Neuroscience Letters, 1985. 58(1): p. 37-41.

56. Kalsbeek, A., et al., In vivo measurement of a diurnal variation in vasopressin release in the rat suprachiasmatic nucleus. Brain Research, 1995. 682(1): p. 75-82.

57. Paszek, P., et al., Population robustness arising from cellular heterogeneity. Proceedings of the National Academy of Sciences, 2010. 107(25): p. 11644.

58. $\mathrm{Gu}, \mathrm{C}$. and $\mathrm{H}$. Yang, Differences in intrinsic amplitudes of neuronal oscillators improve synchronization in the suprachiasmatic nucleus. Chaos: An Interdisciplinary Journal of Nonlinear Science, 2017. 27(9): p. 093108.

59. Enoki, R., et al., Synchronous circadian voltage rhythms with asynchronous calcium rhythms in the suprachiasmatic nucleus. Proceedings of the National Academy of Sciences, 2017. 114(12): p. E2476.

60. Pauls, S., et al., Differential contributions of intra-cellular and inter-cellular mechanisms to the spatial and temporal architecture of the suprachiasmatic nucleus circadian circuitry in wild-type, cryptochrome-null and vasoactive intestinal peptide receptor 2-null mutant mice. European Journal of Neuroscience, 2014. 40(3): p. 2528-2540.

61. Bush, W.S. and H.T. Siegelman, Circadian synchrony in networks of protein rhythm driven neurons. Complexity, 2006. 12(1): p. 67-72.

62. Wagner, S., et al., GABA in the mammalian suprachiasmatic nucleus and its role in diurnal rhythmicity. Nature, 1997. 387(6633): p. 598-603.

63. Choi, H.J., et al., Excitatory Actions of GABA in the Suprachiasmatic Nucleus. The Journal of Neuroscience, 2008. 28(21): p. 5450-5459.

64. Jeu, M.D. and C. Pennartz, Circadian Modulation of GABA Function in the Rat Suprachiasmatic Nucleus: Excitatory Effects During the Night Phase. Journal of Neurophysiology, 2002. 87(2): p. 834-844.

65. Irwin, R.P. and C.N. Allen, GABAergic signaling induces divergent neuronal Ca2+ responses in the suprachiasmatic nucleus network. European Journal of Neuroscience, 2009. 30(8): p. 1462-1475.

66. McNeill, J.K., J.C. Walton, and H.E. Albers, Functional Significance of the Excitatory Effects of GABA in the Suprachiasmatic Nucleus. Journal of Biological Rhythms, 2018. 33(4): p. 376-387. 

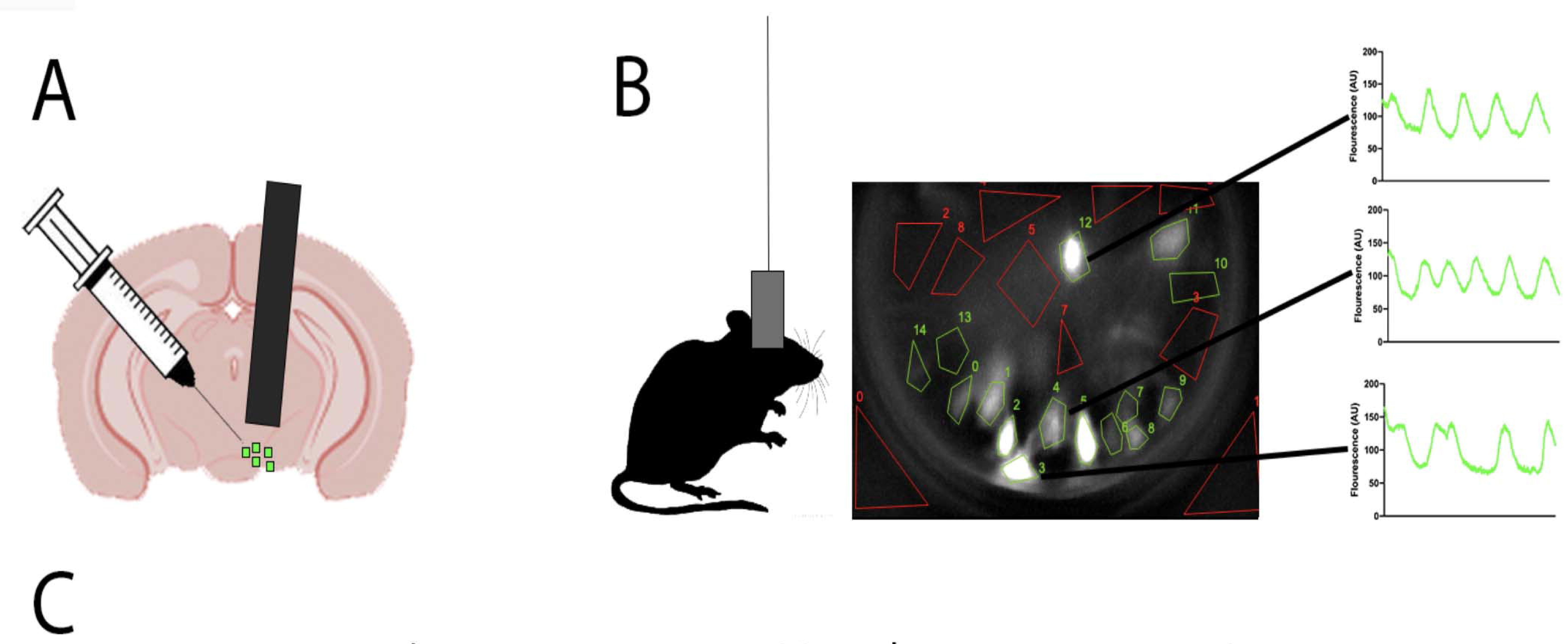

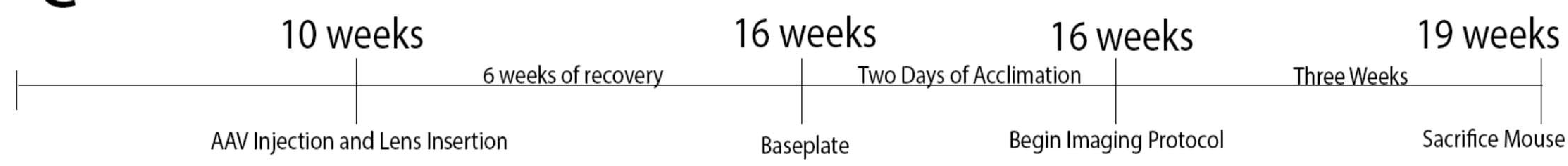
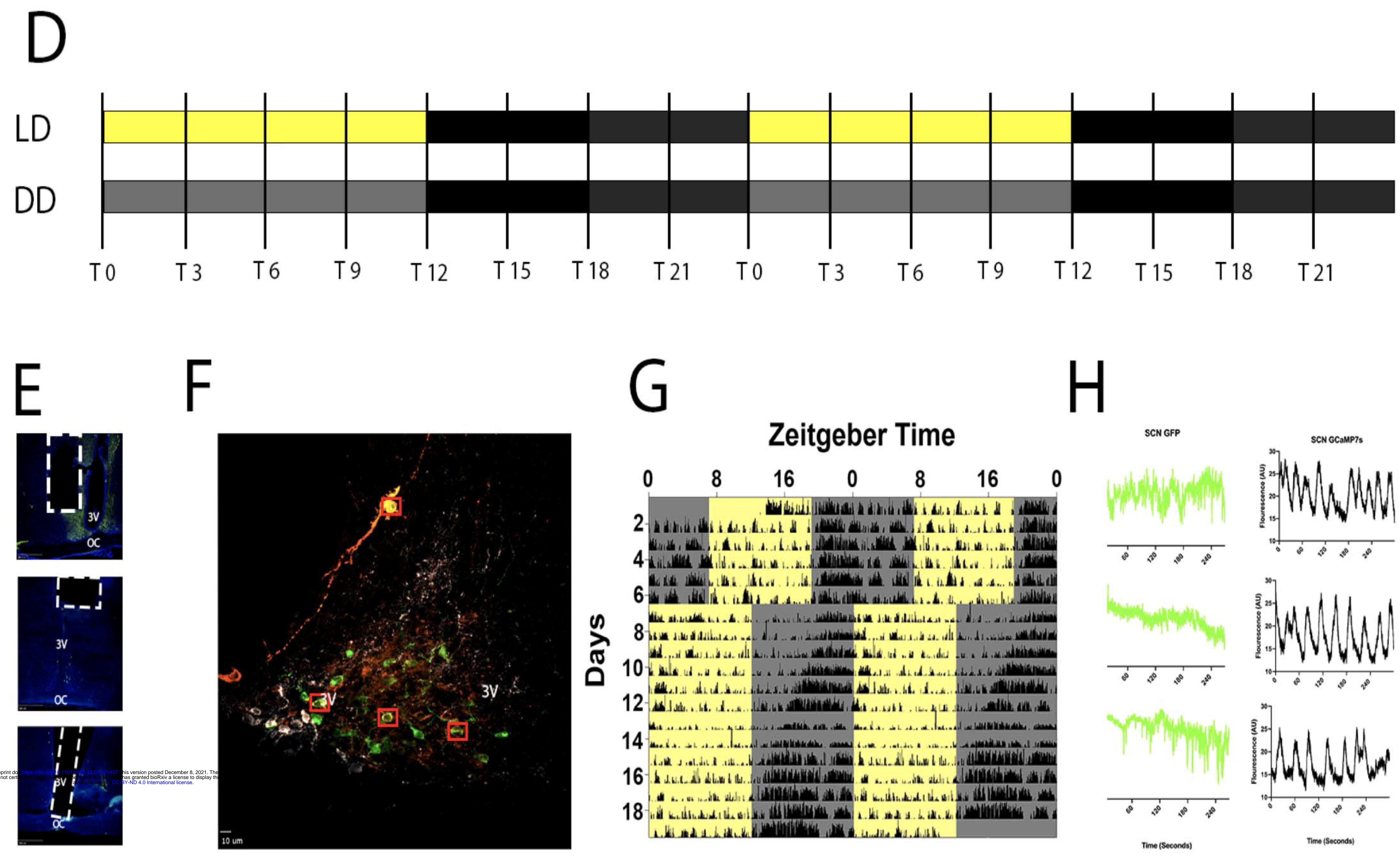
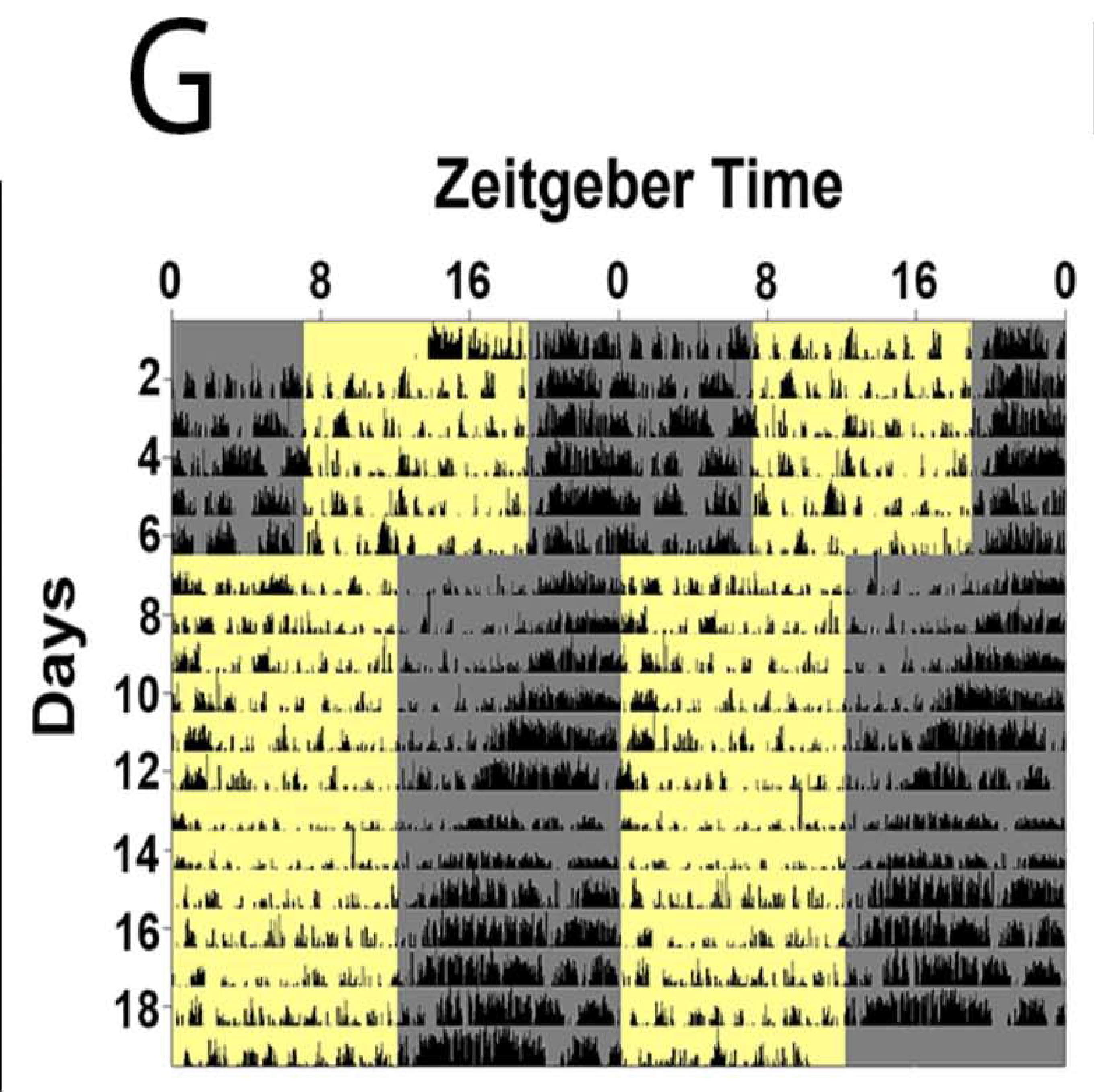

$\mathrm{H}$

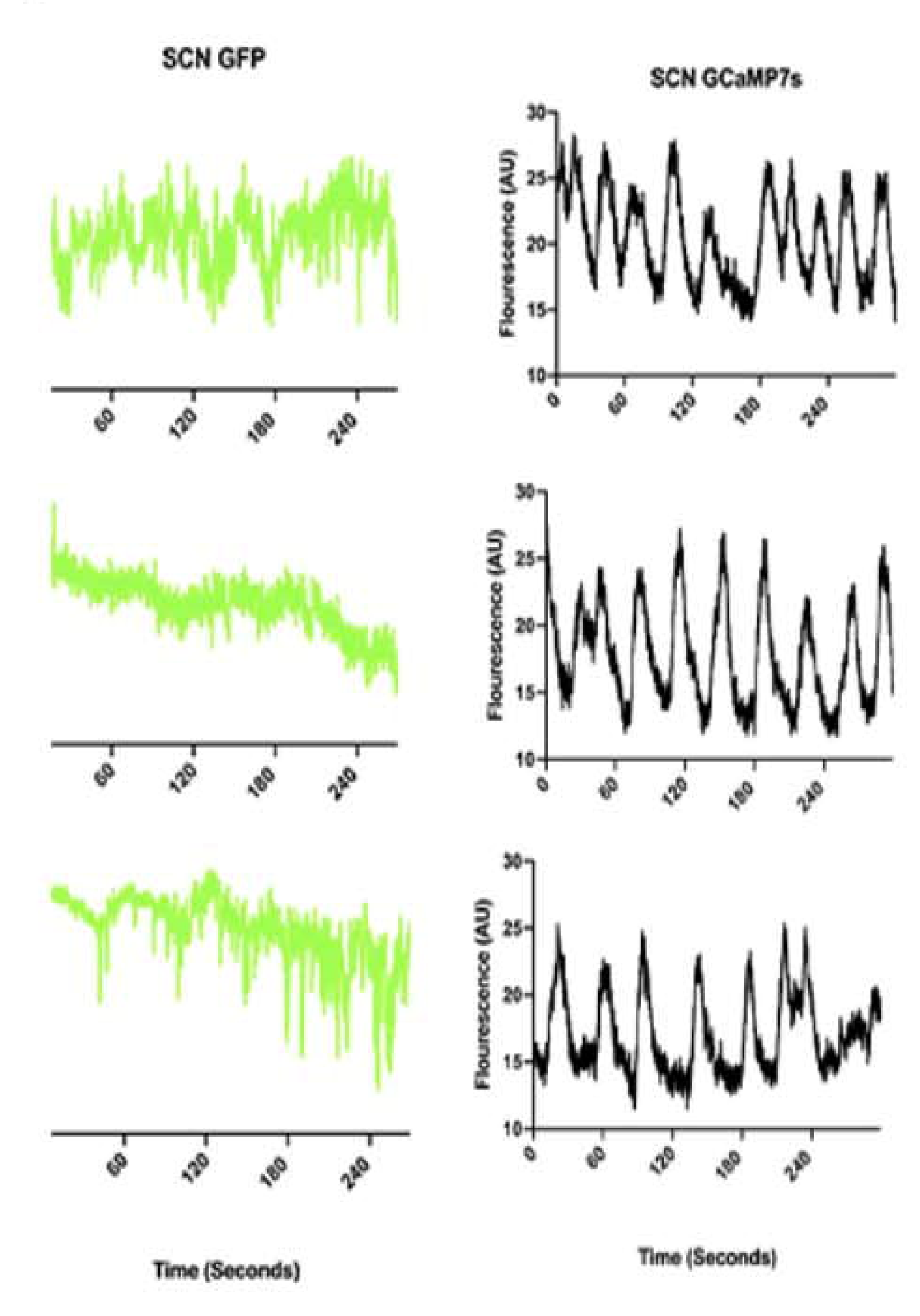




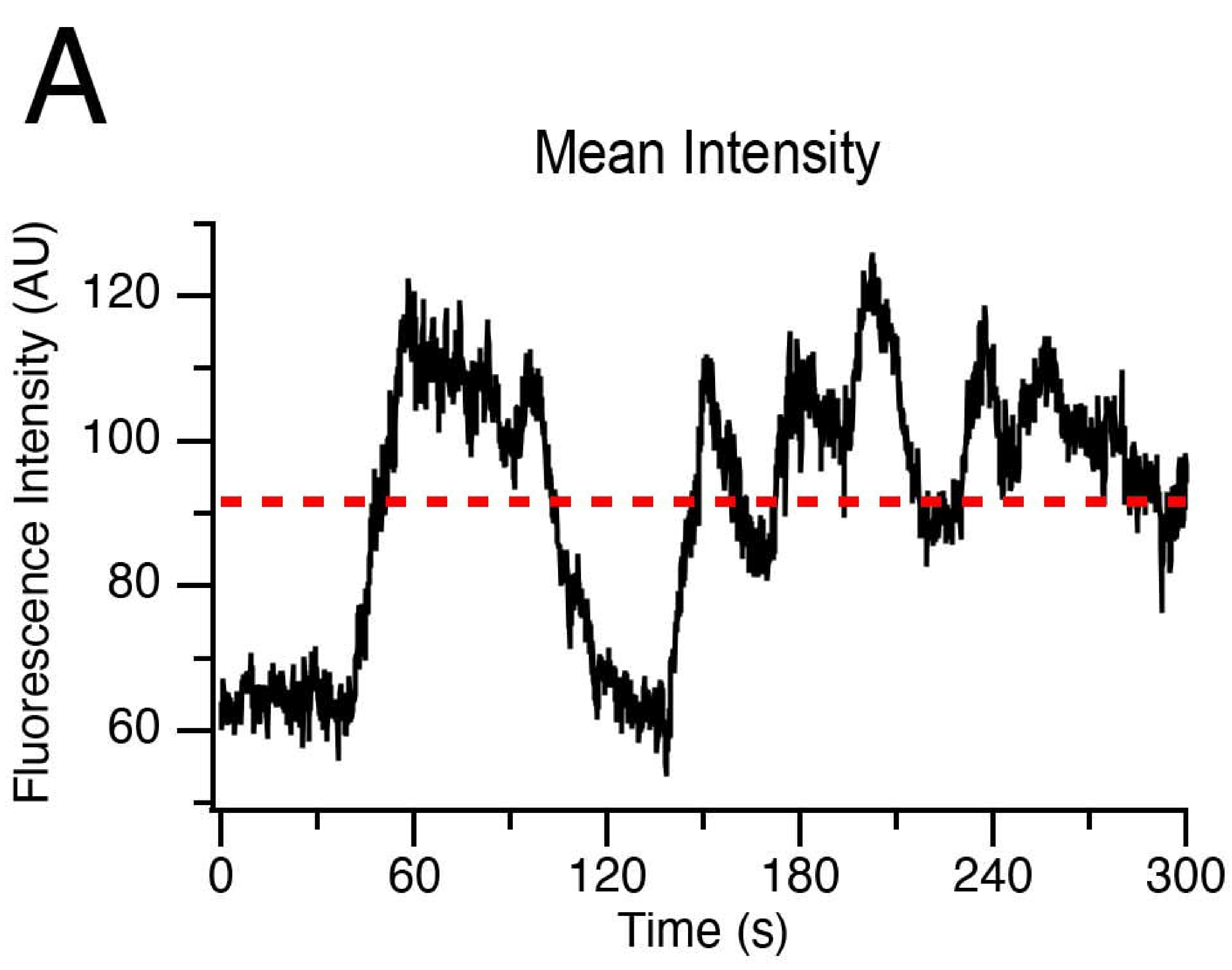

B

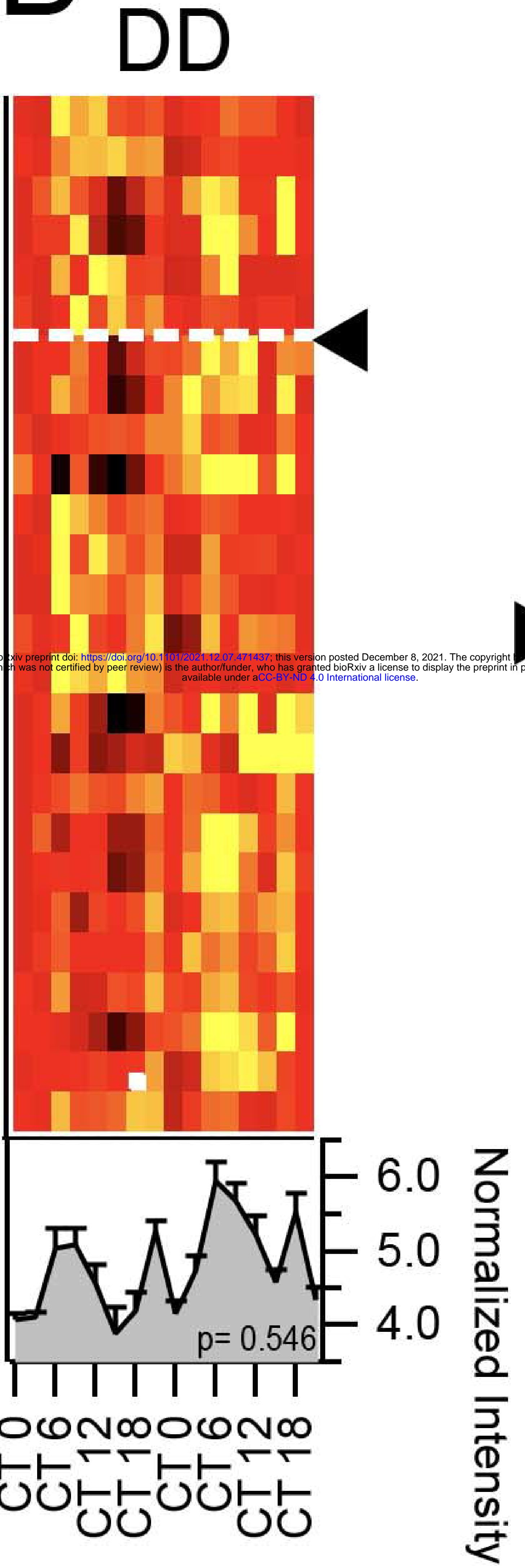

\section{LD}

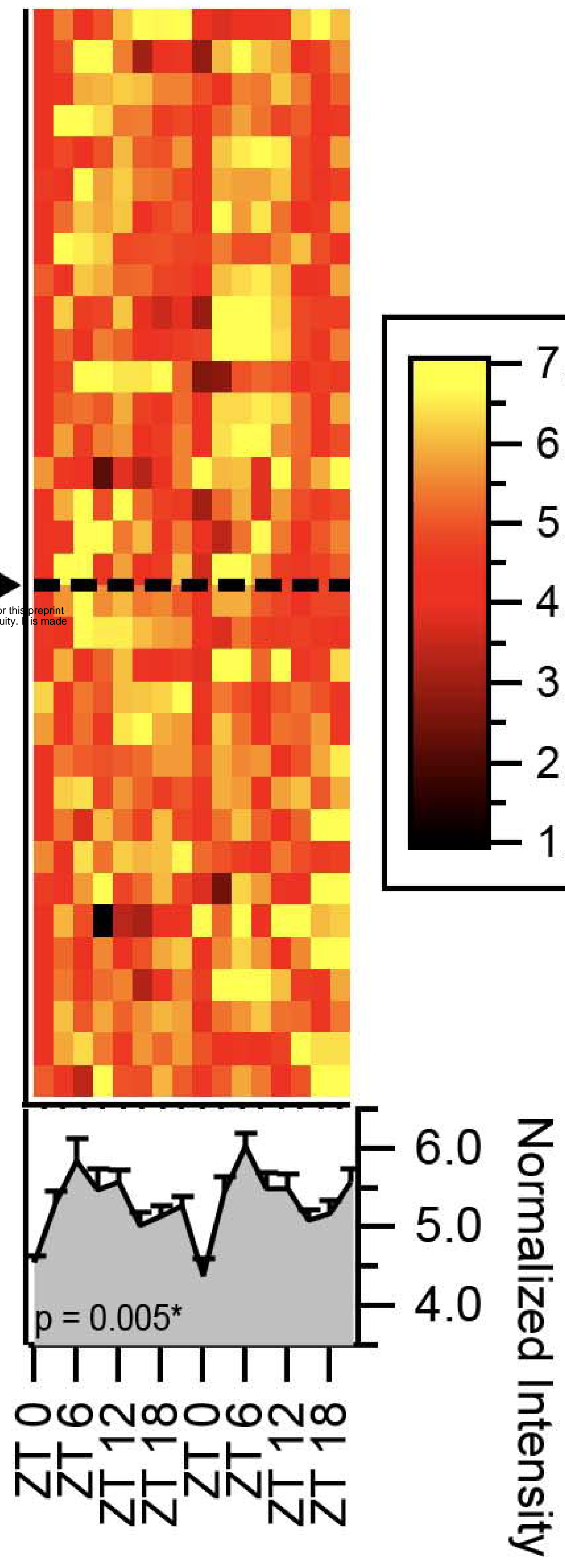

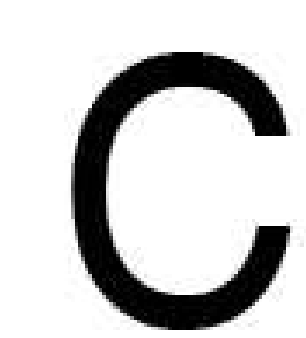
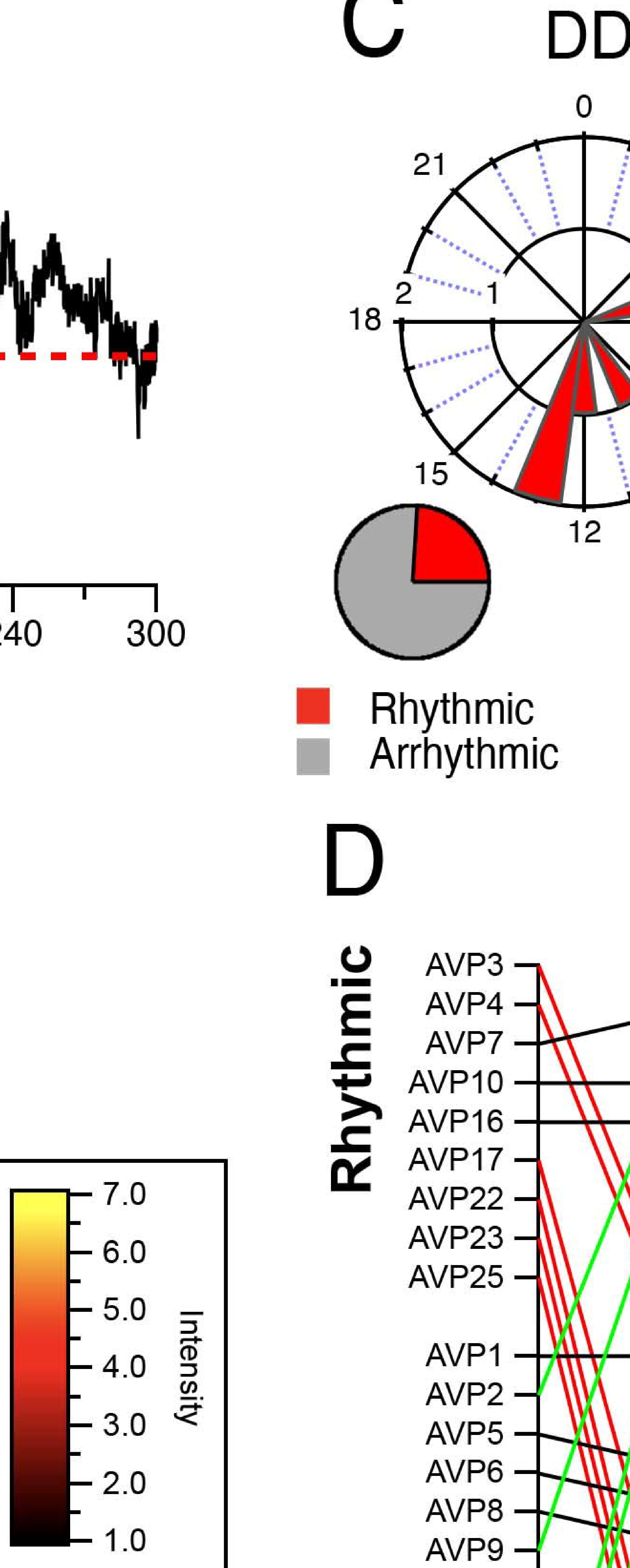

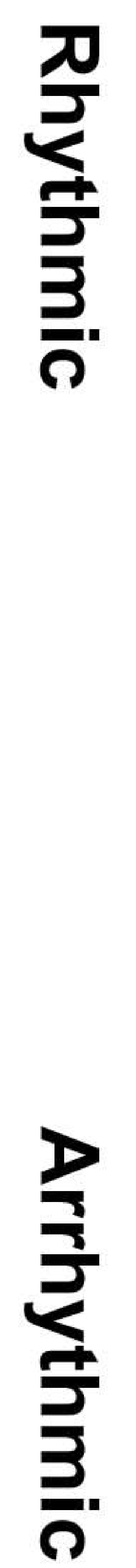

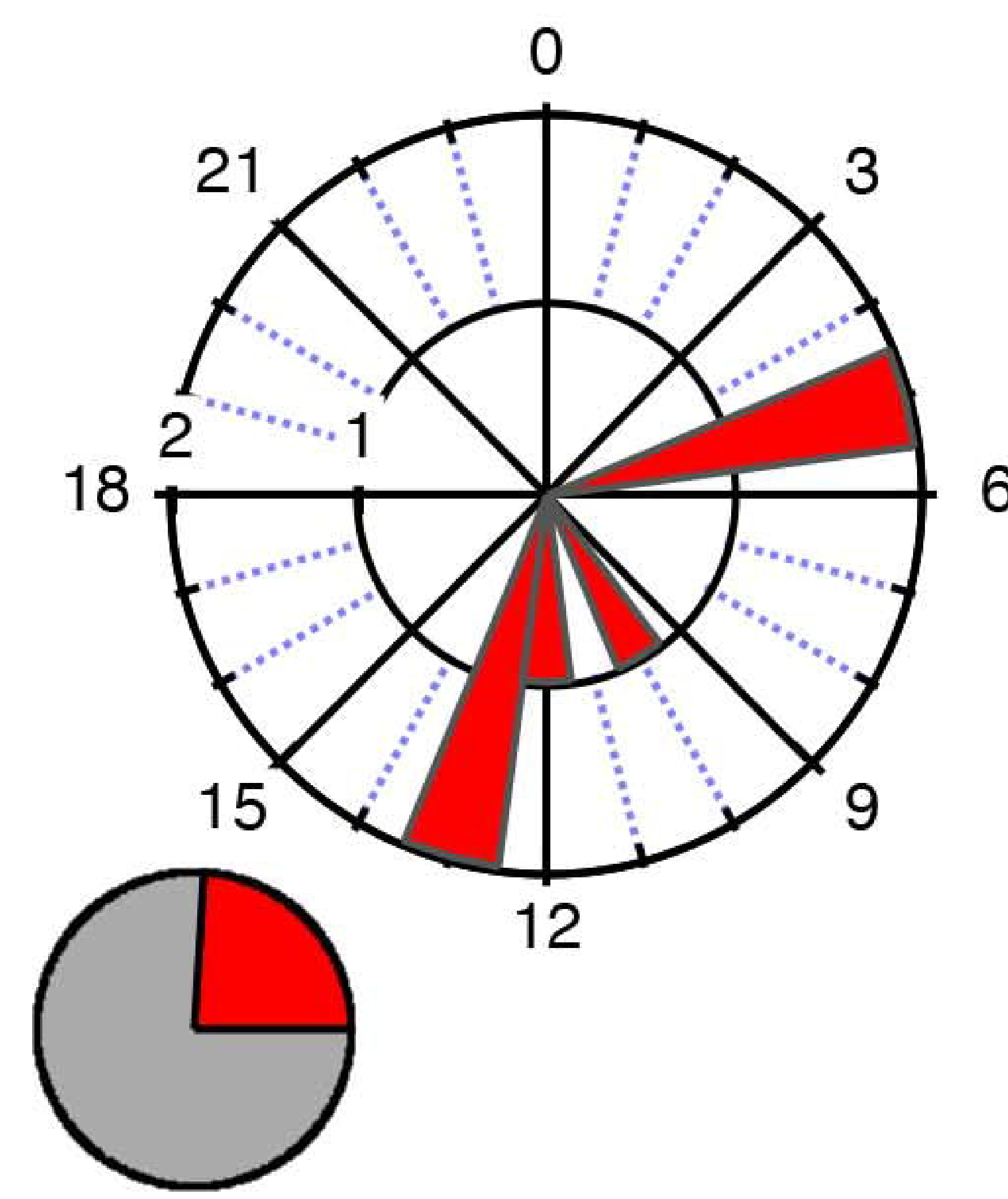

Rhythmic

Arrhythmic

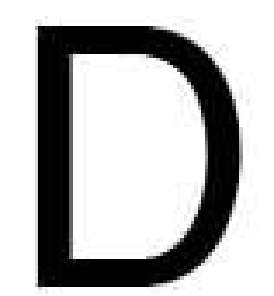

$\frac{\underline{n}}{\frac{E}{2}}$

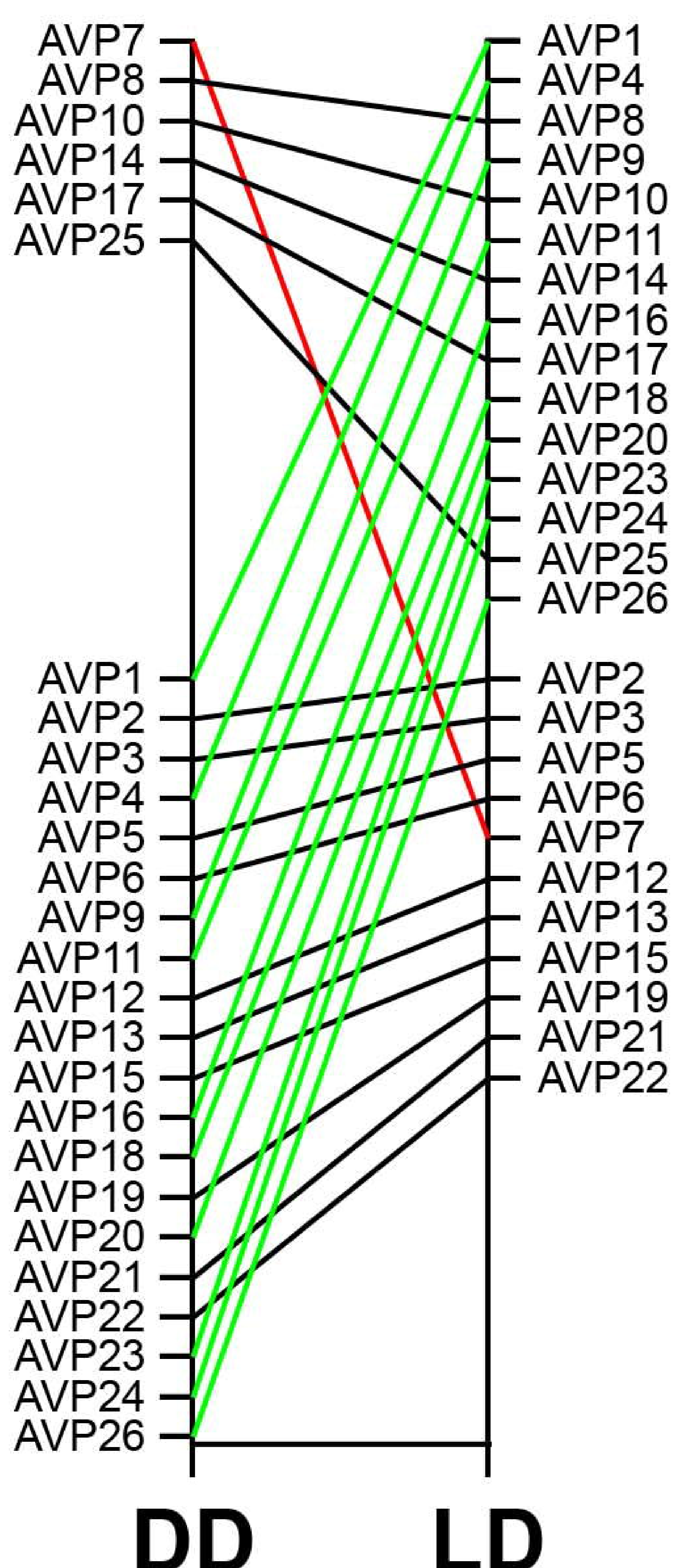

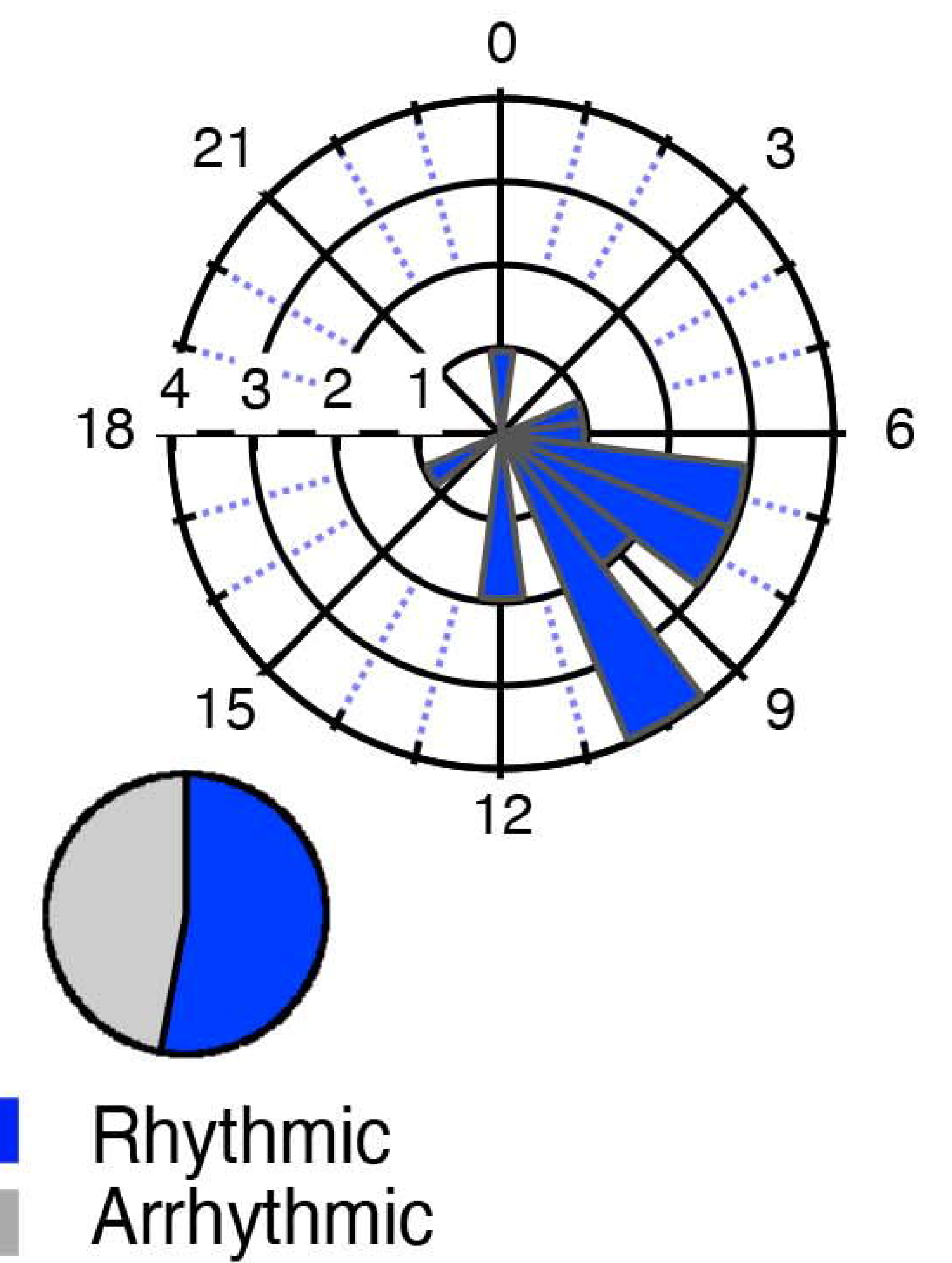

)

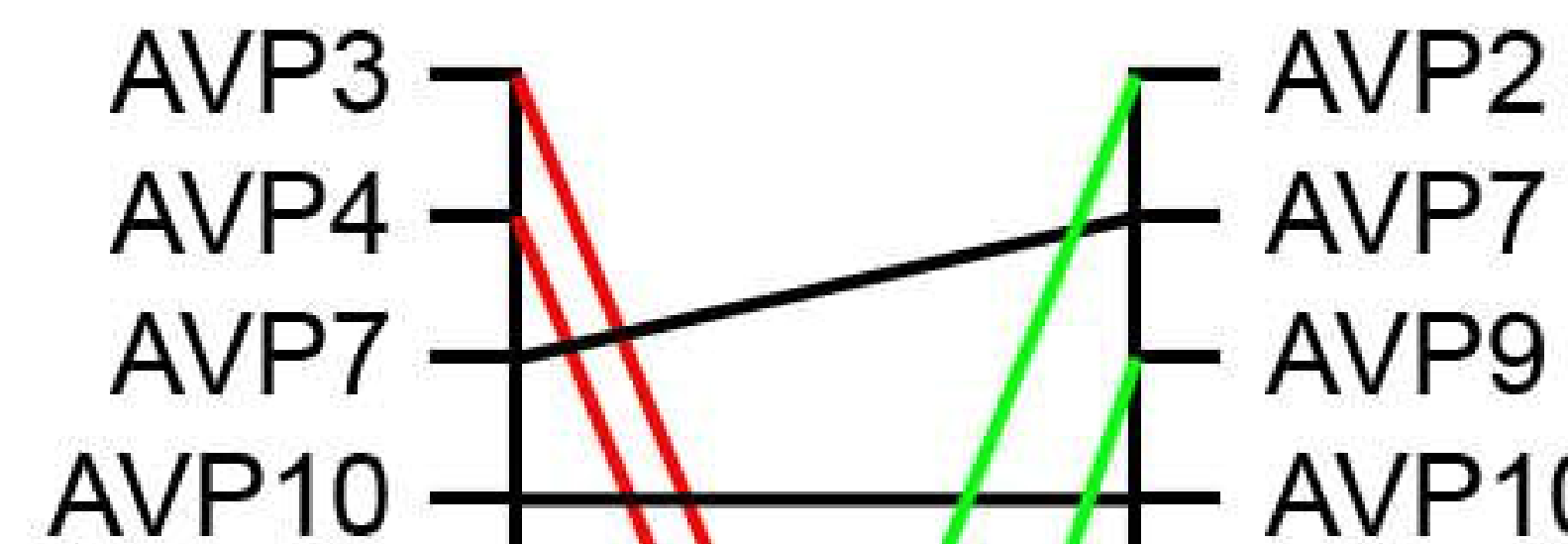

AVP16 $12-2$ AVP16

AVP17- - -

AVP22 - - AVP19

AVP23 - AVP24

AVP25 -

AVP1 - AVP - AVP

AVP2 -

AVP5 - 1 - AVP4

AVP6 $-A V P 5$

AVP8 $-11-$ AVP6

AVP9 AVP8

AVP11 - AVP11

AVP12 - $-A V P 12$

AVP13 - $-A V P 13$

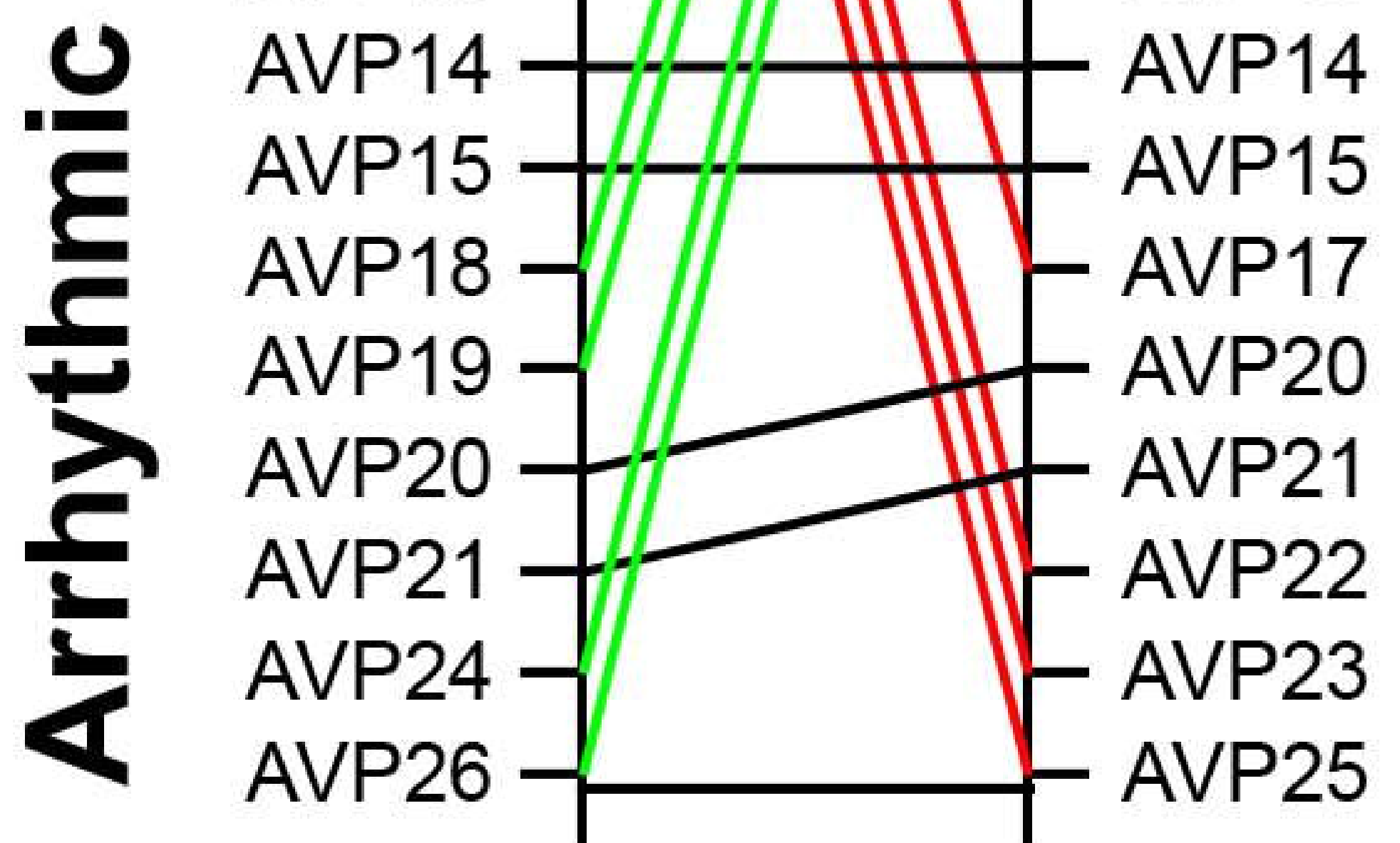

DD1 DD2 

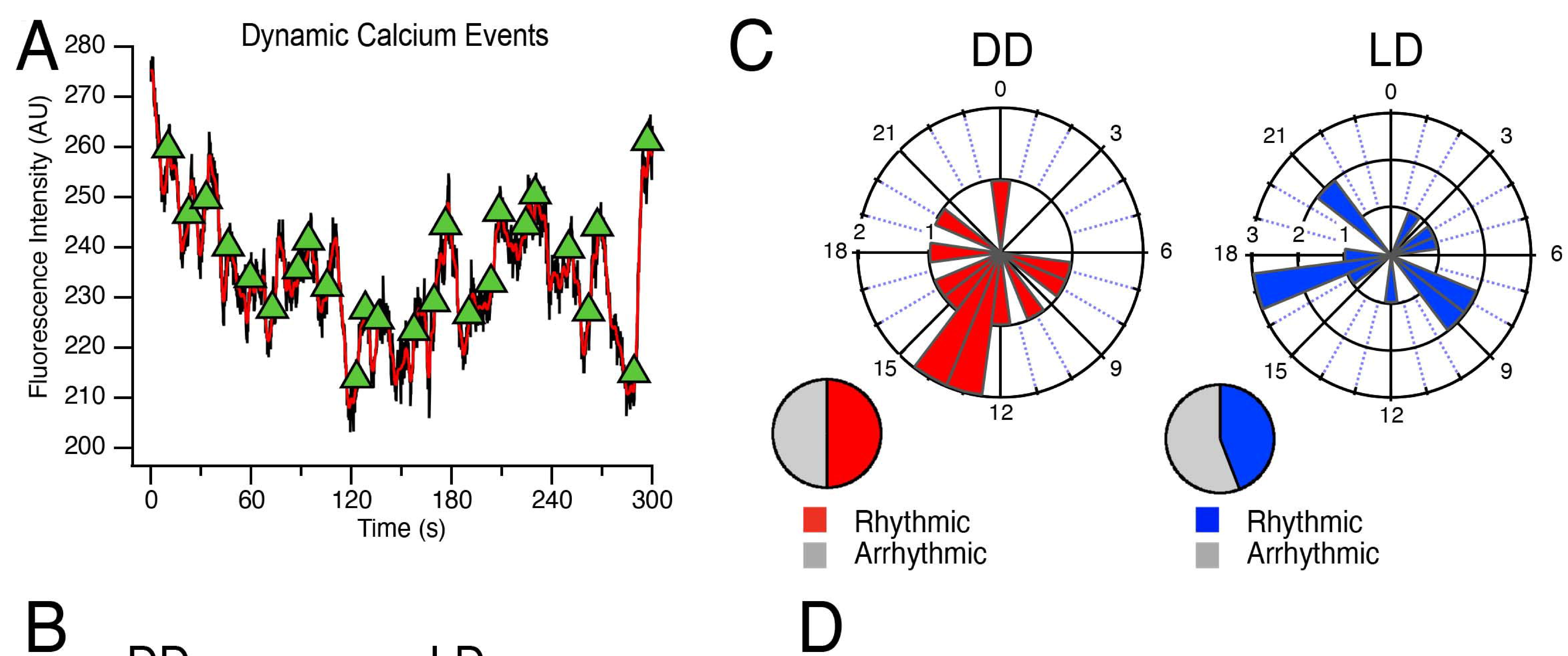

DD

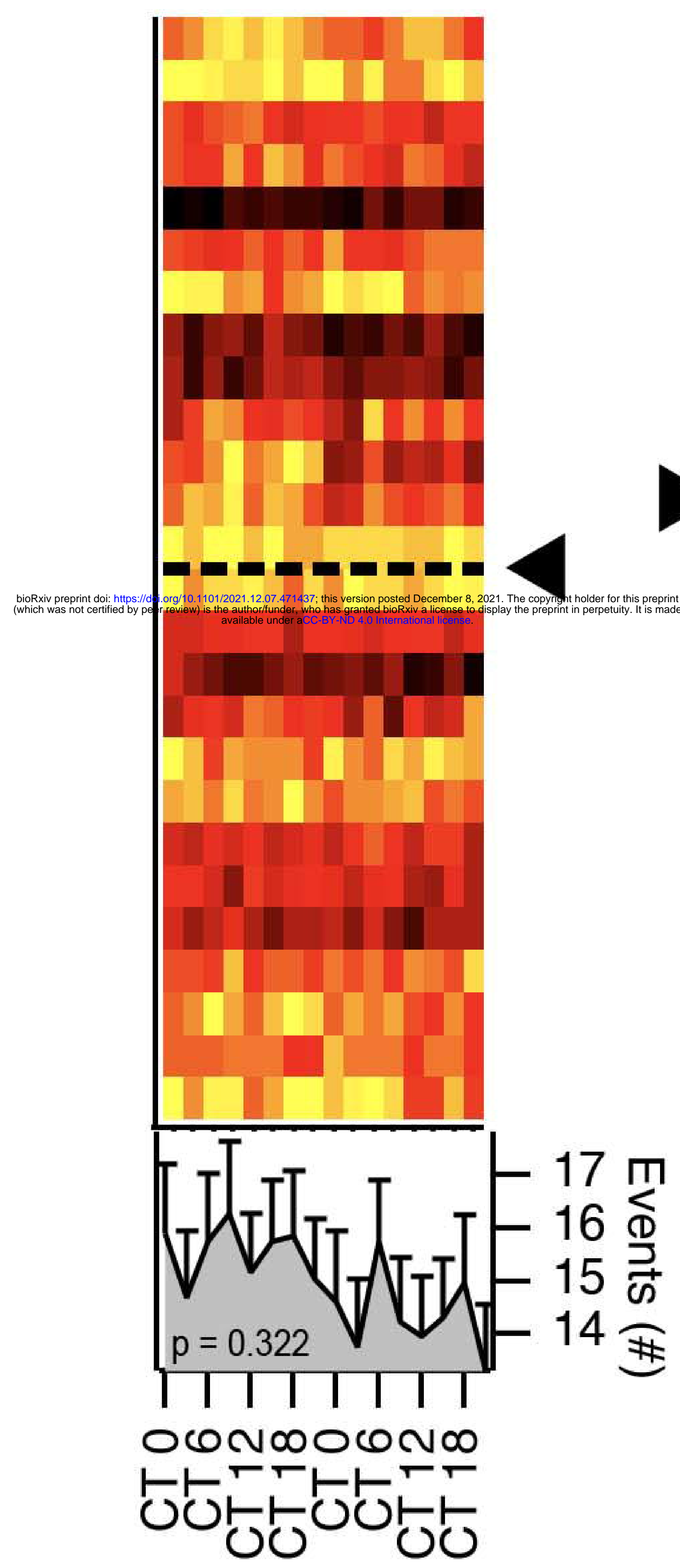

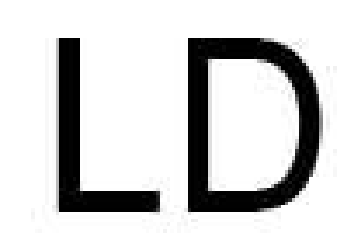

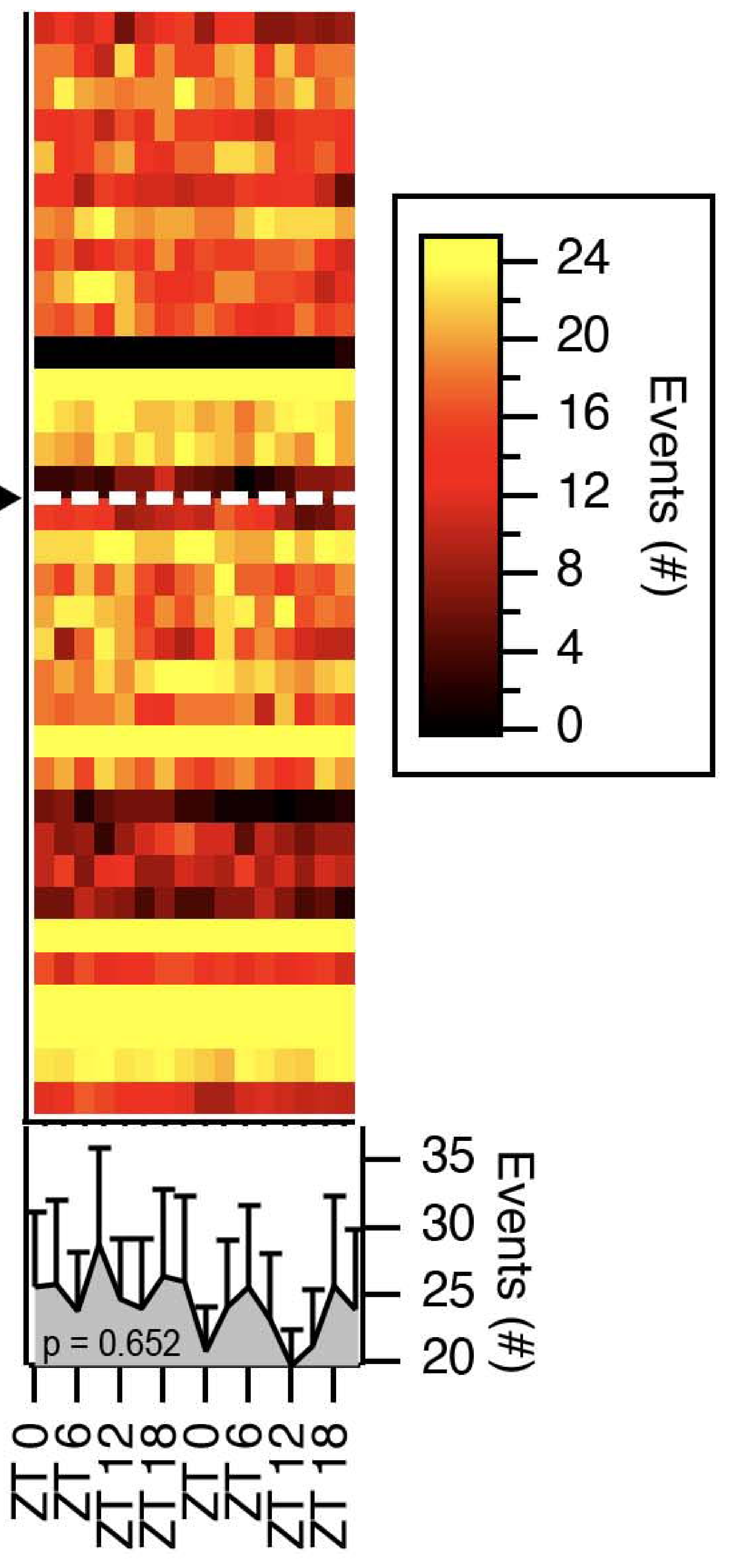

- Rhythmic Arrhythmic
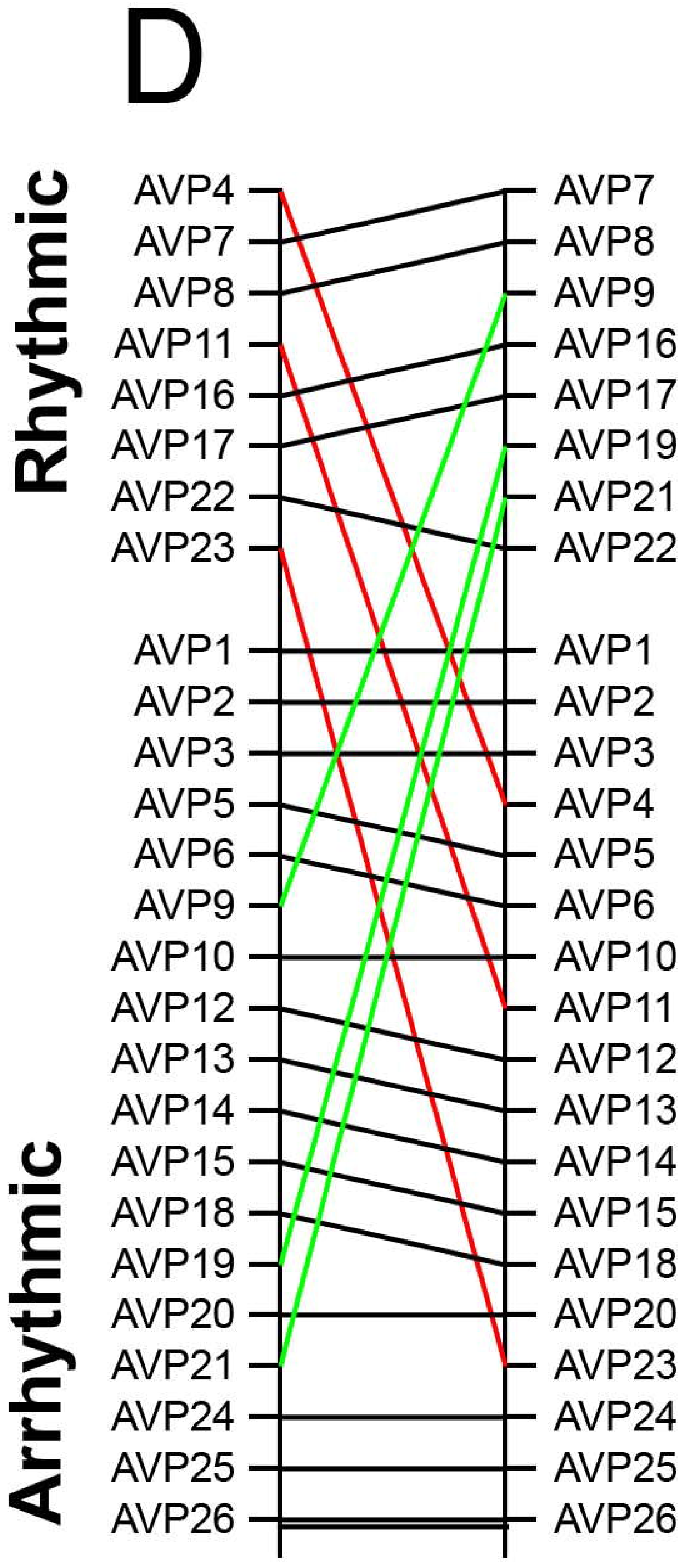

DD1 DD2

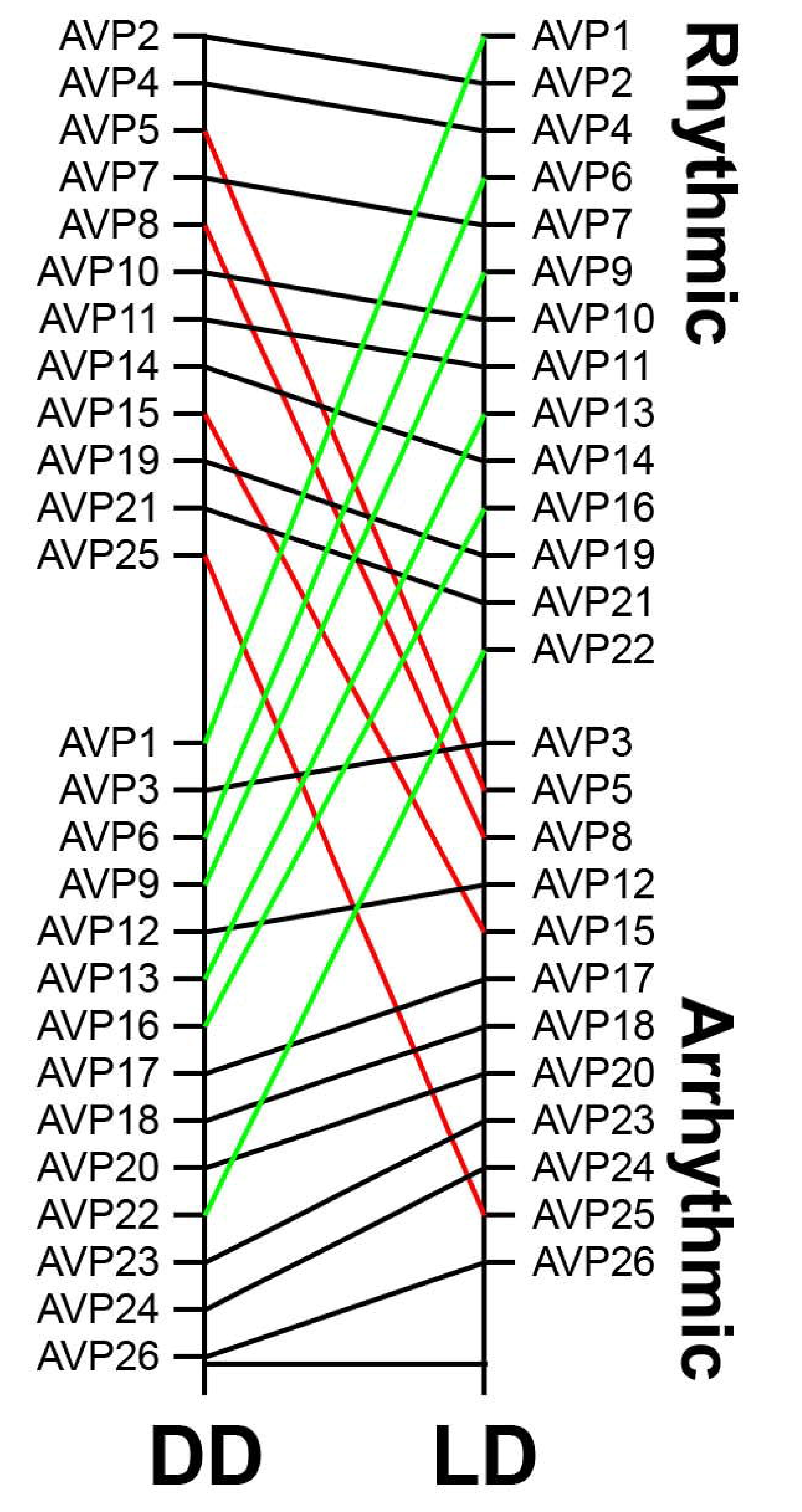



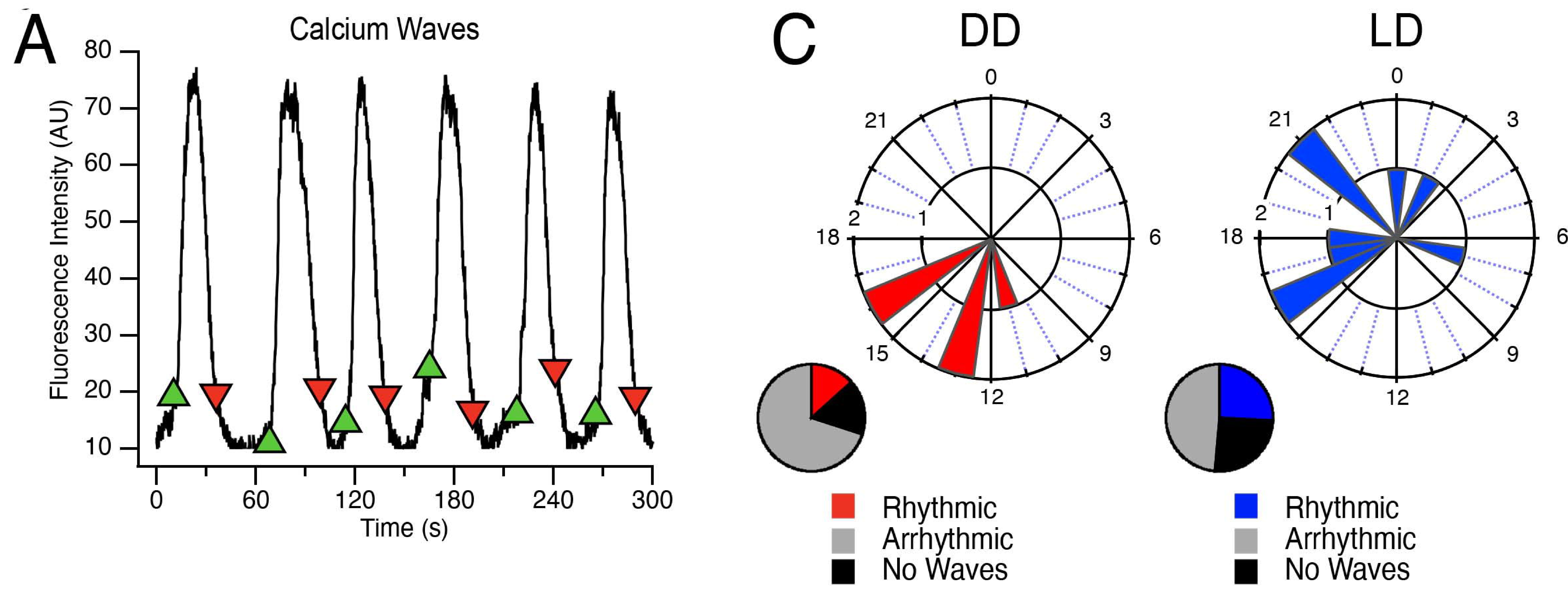

B

DD

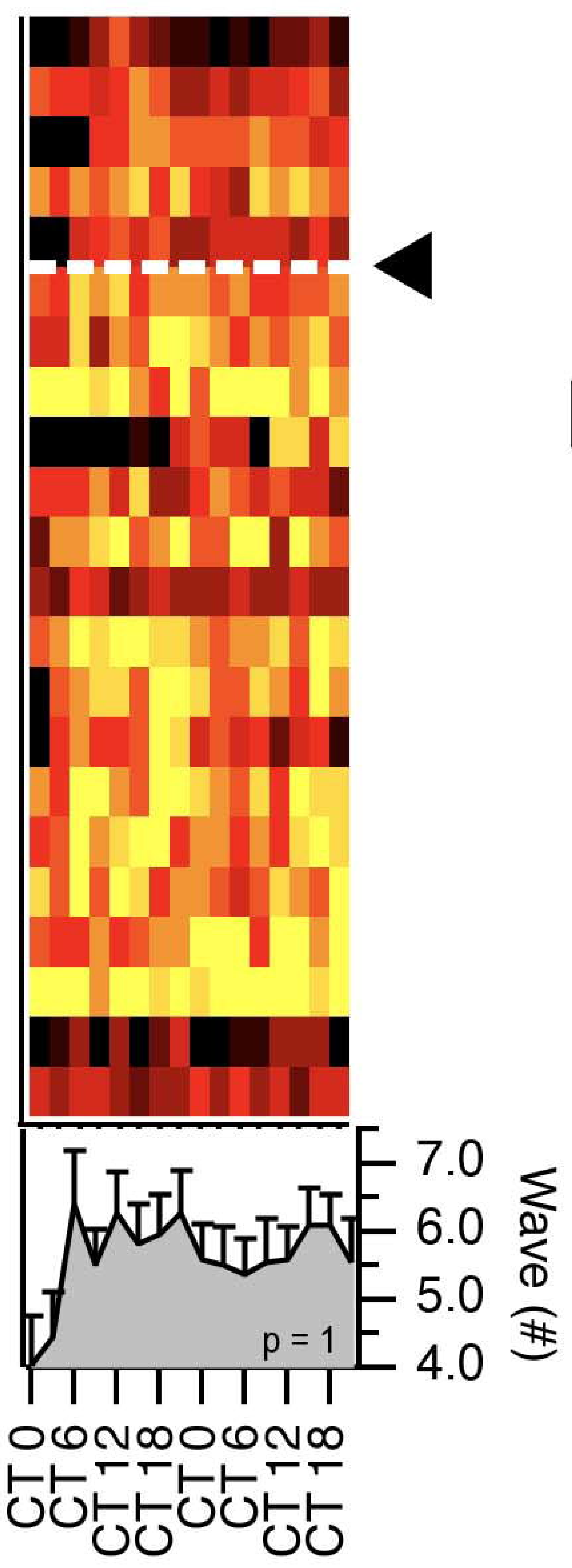

LD

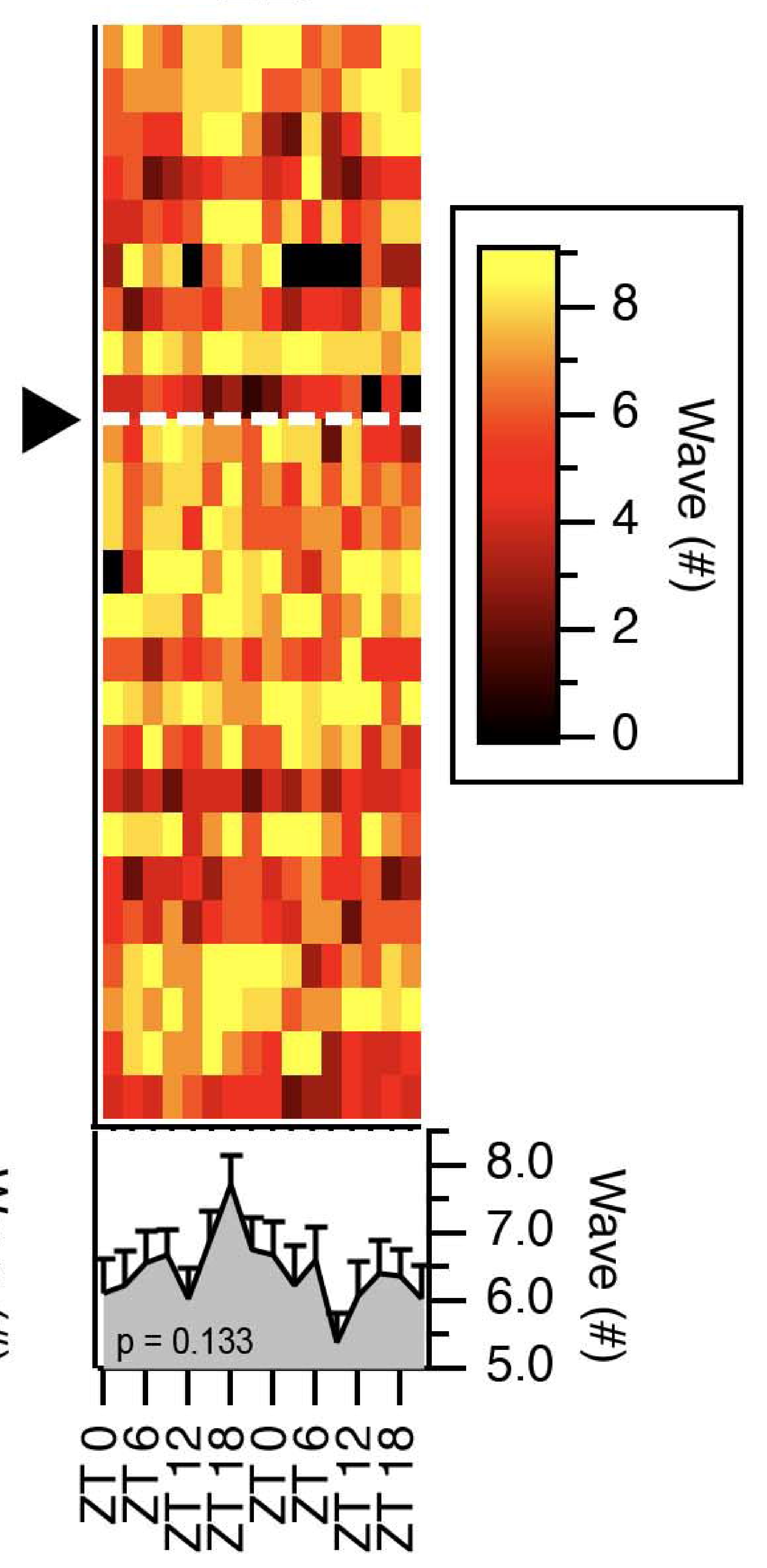

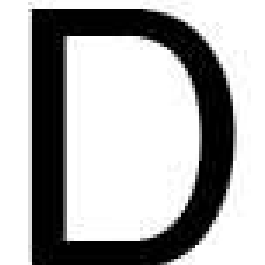

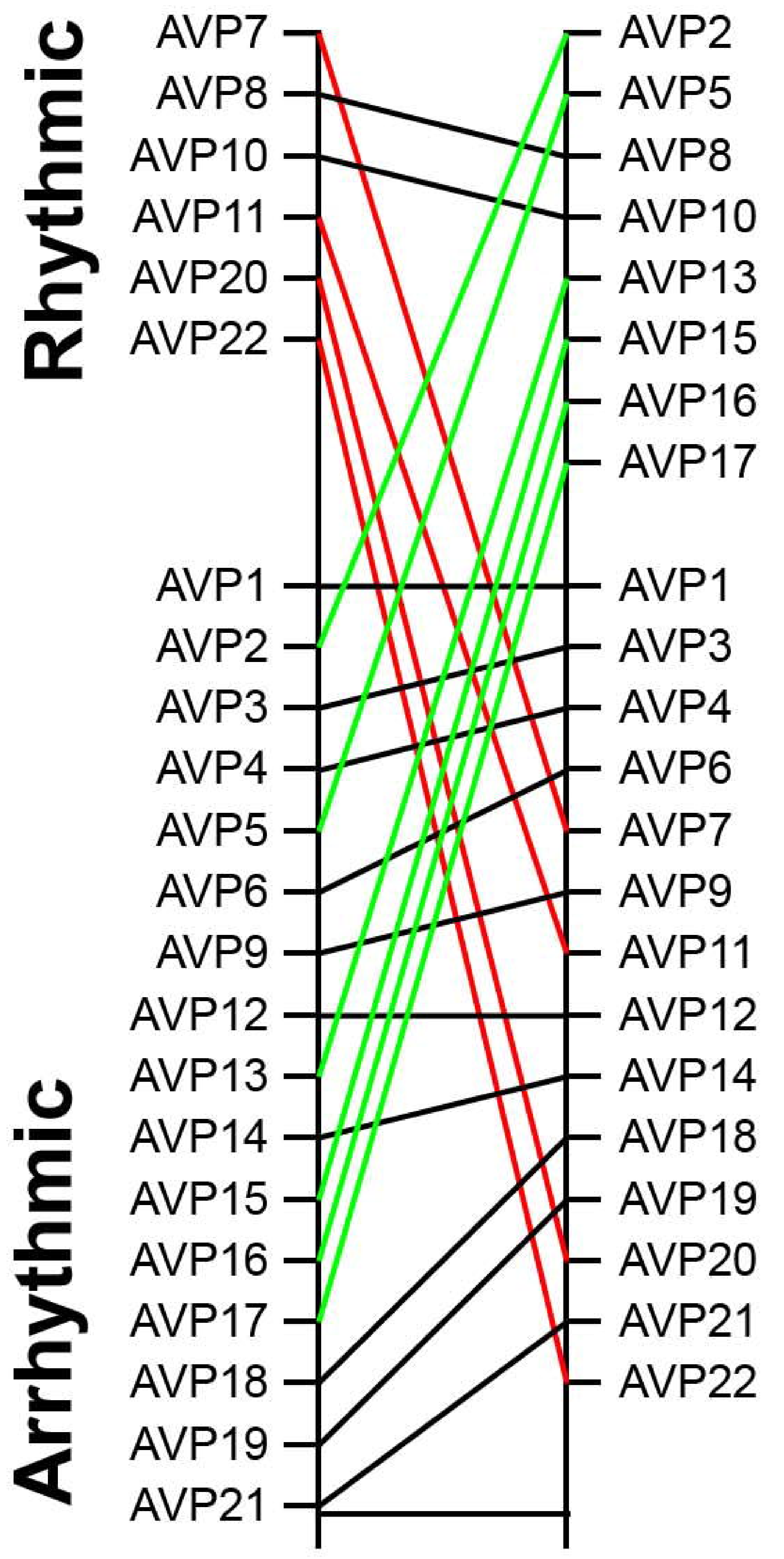

DD1 DD2

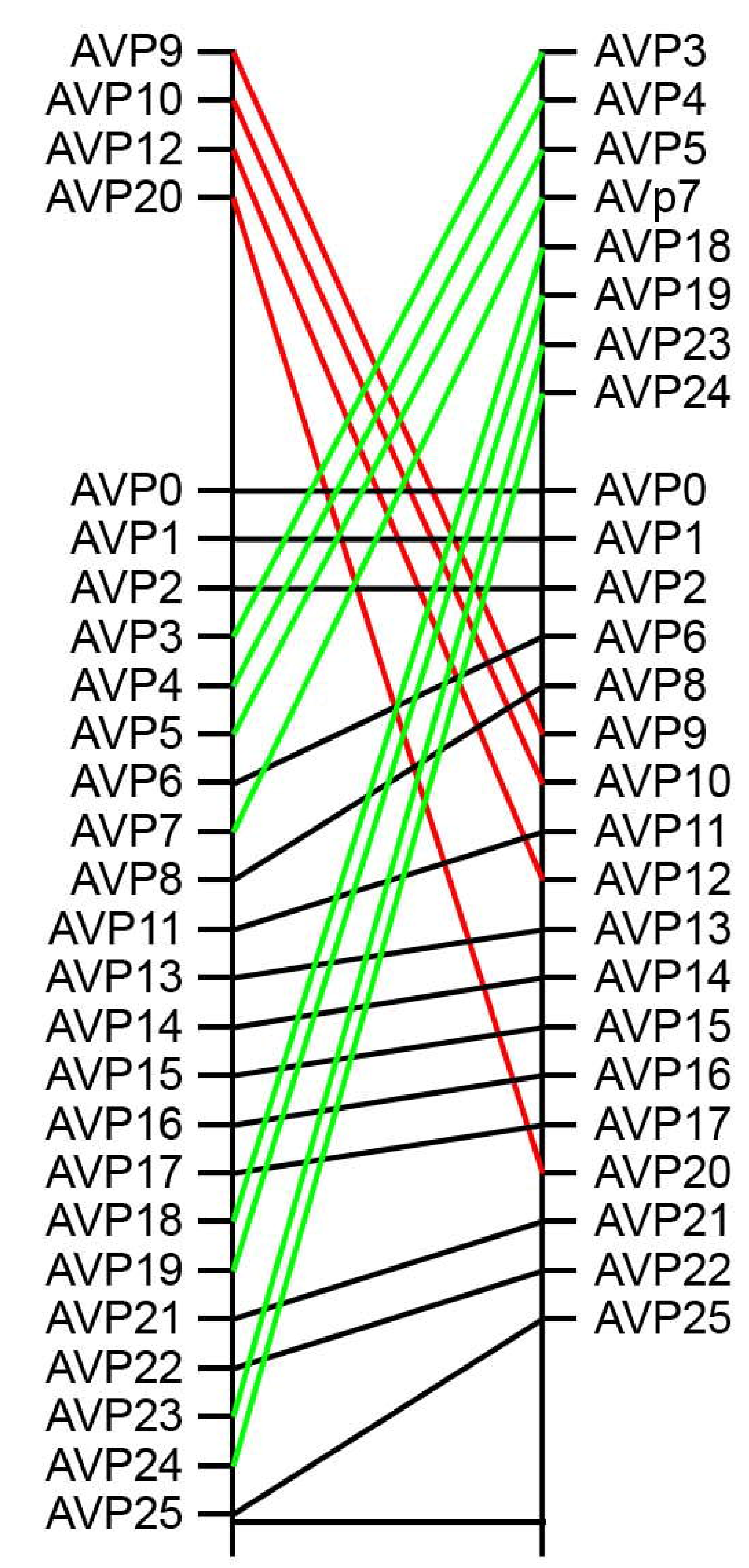

$\frac{\frac{0}{2}}{\frac{3}{3}}$

DD 
A
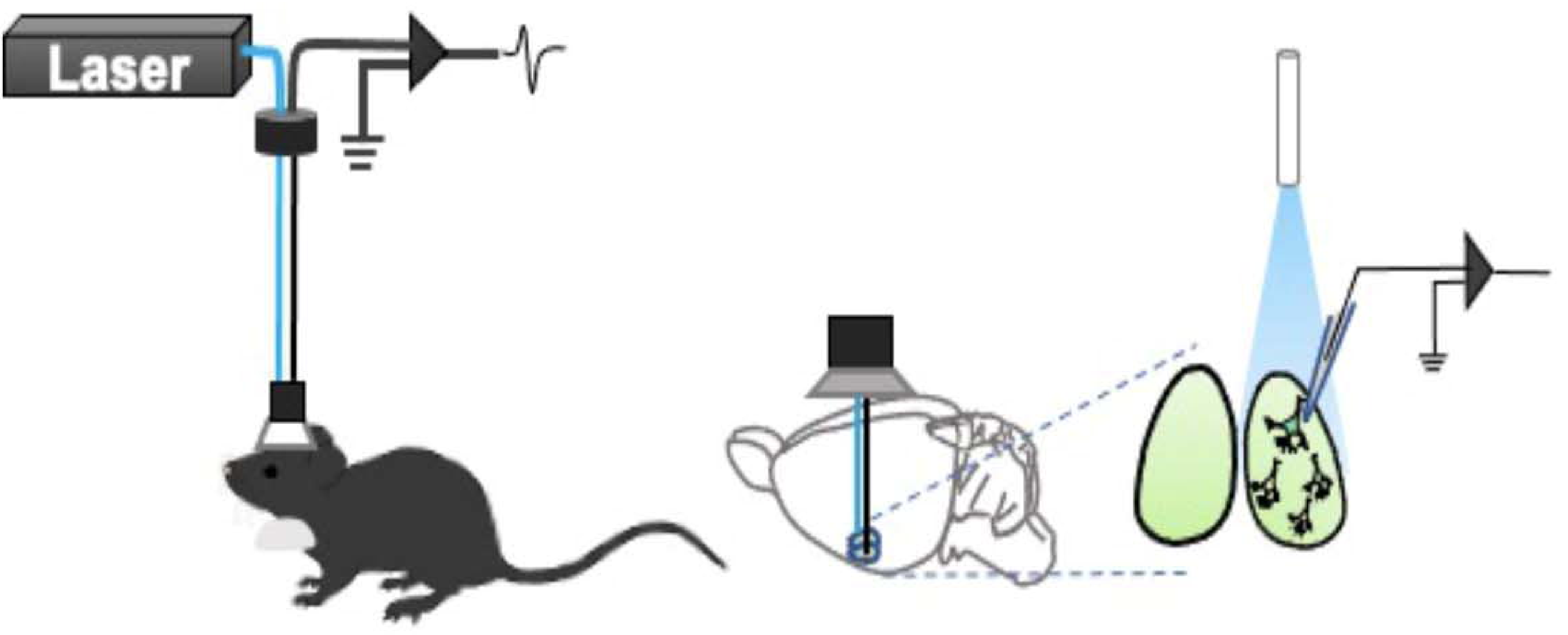

C

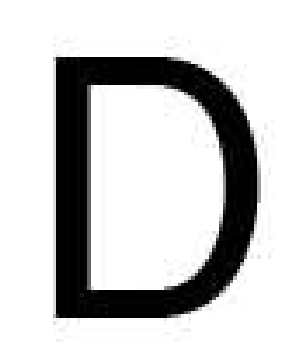

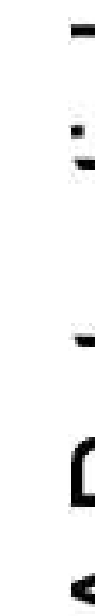
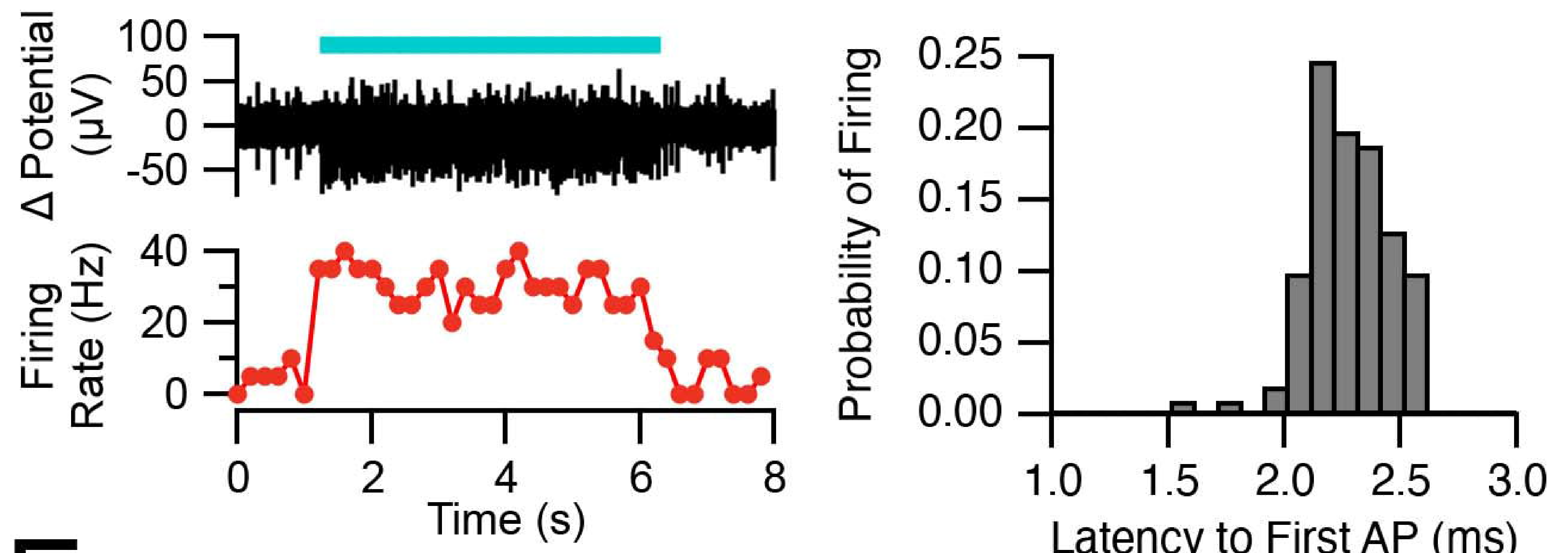

$E$

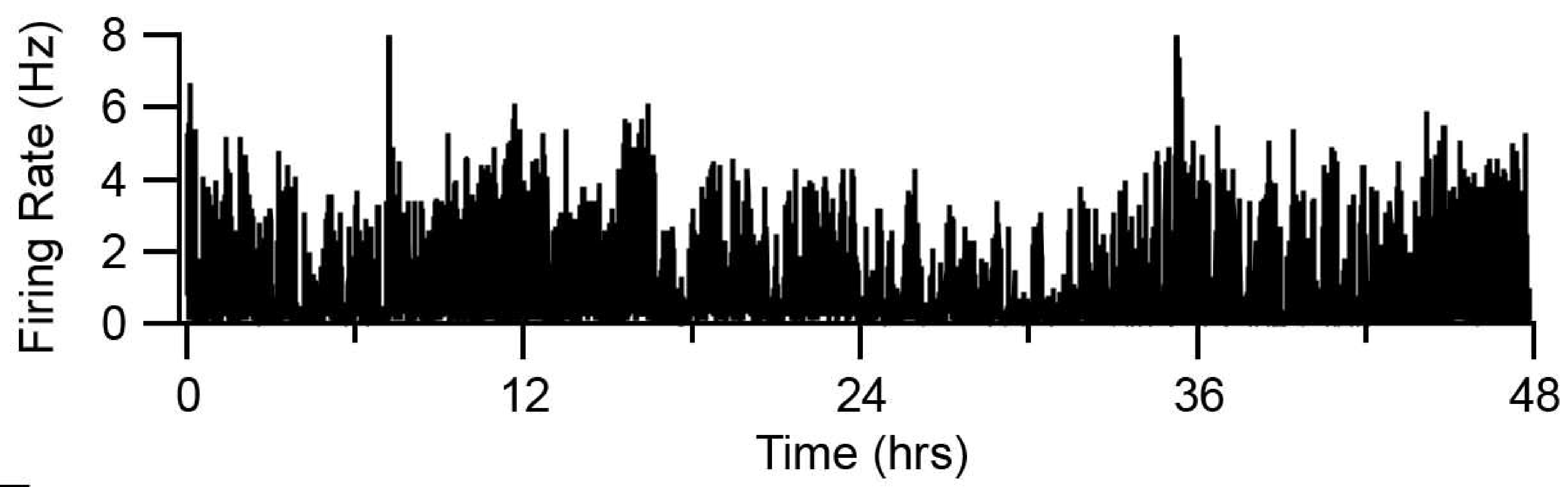

$\mathrm{F}$

OSAR

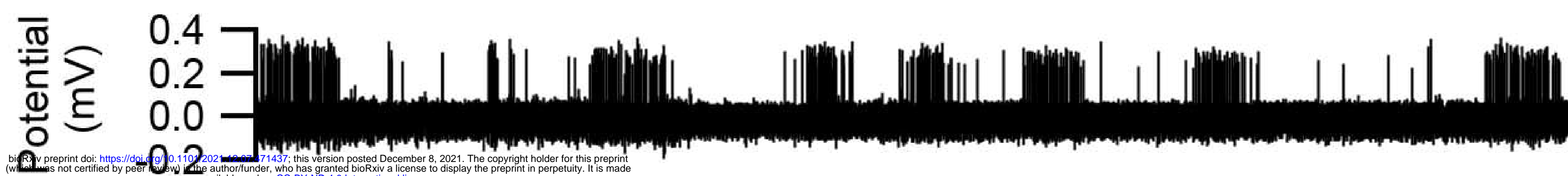

施

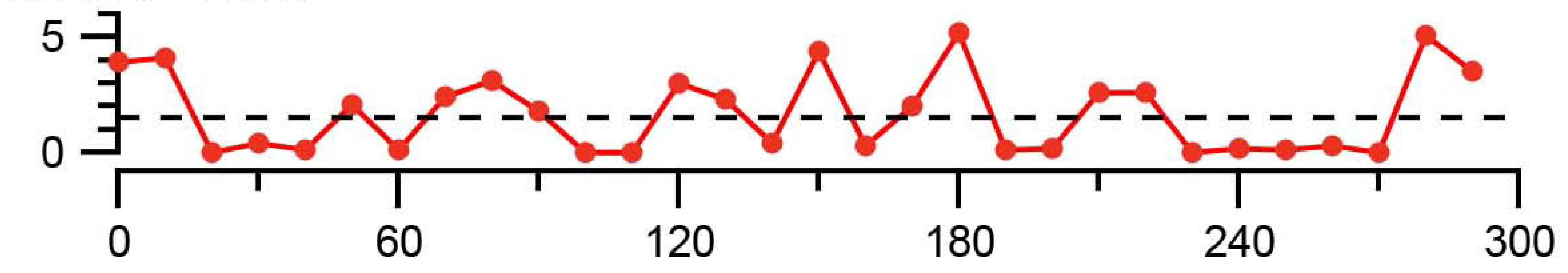

G

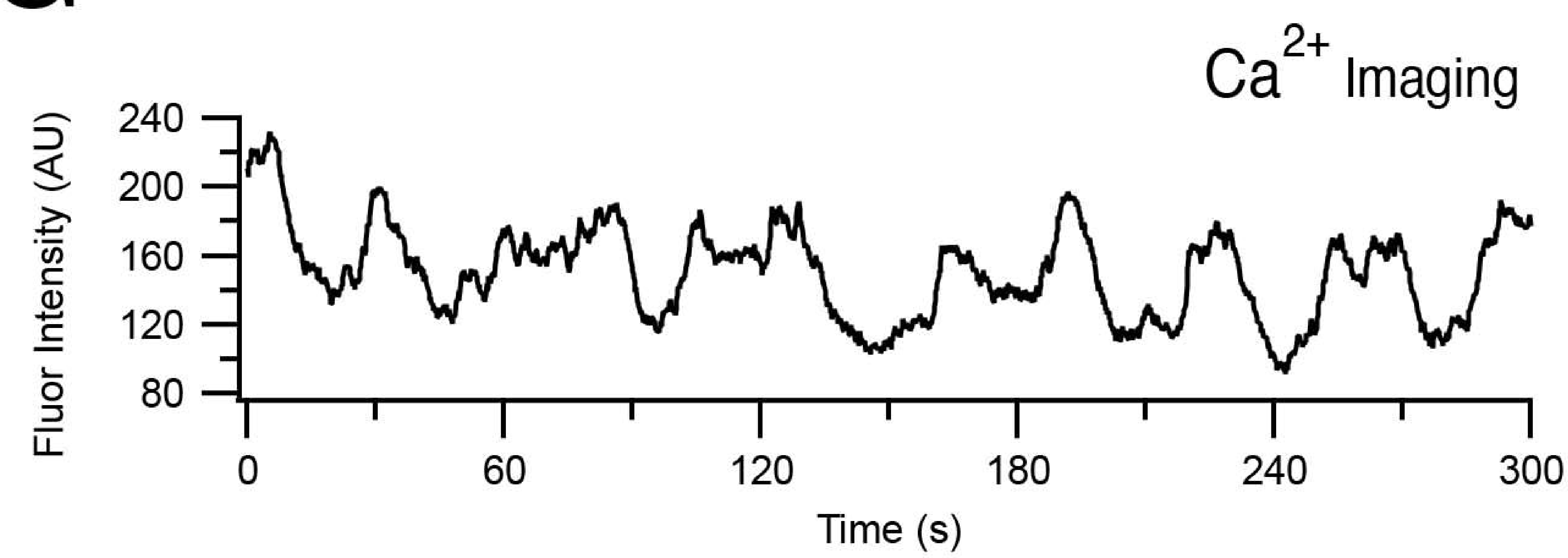

B
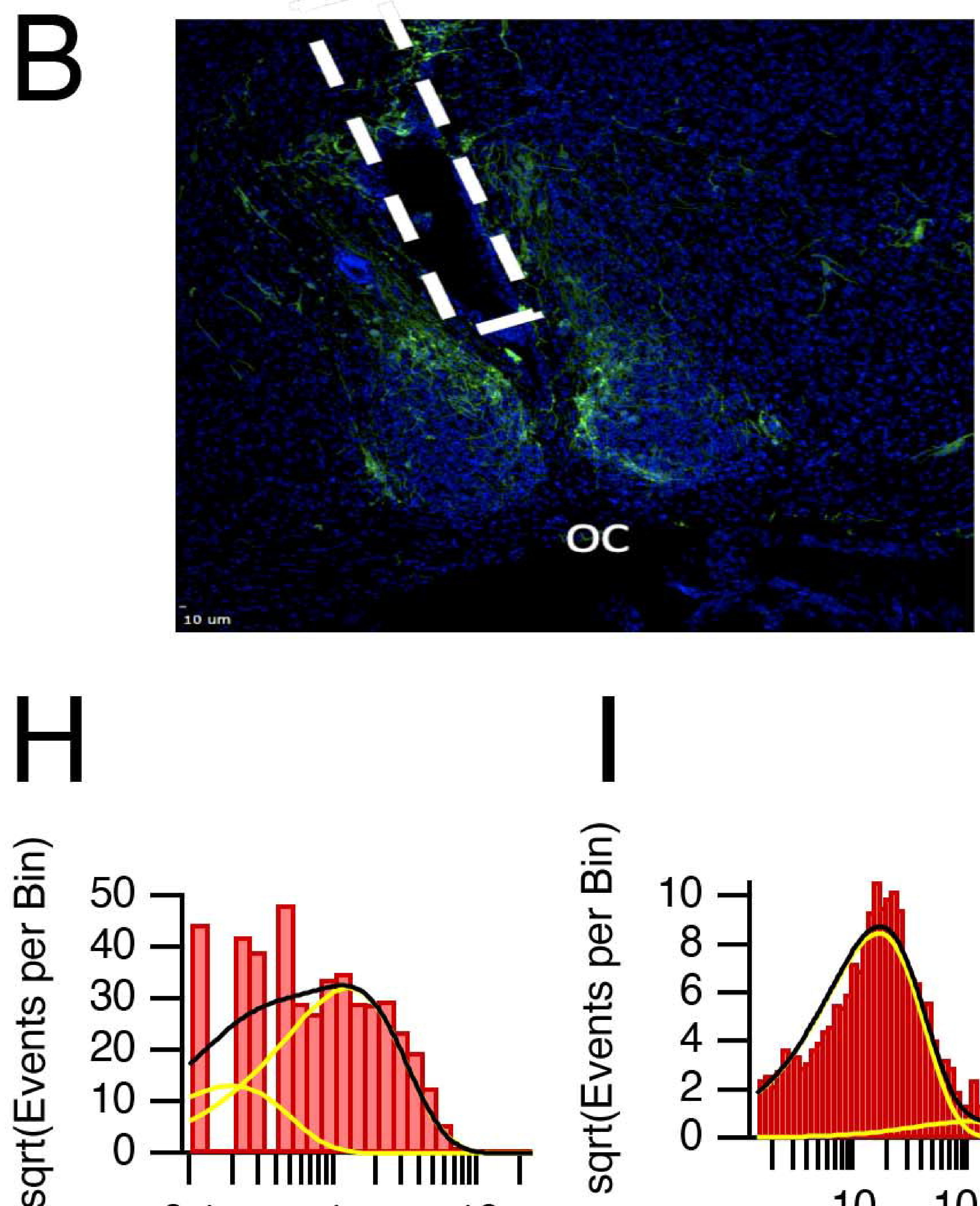

$\begin{array}{lll}0.1 & 1 & 10\end{array}$ Firing Rate $(\mathrm{Hz})$
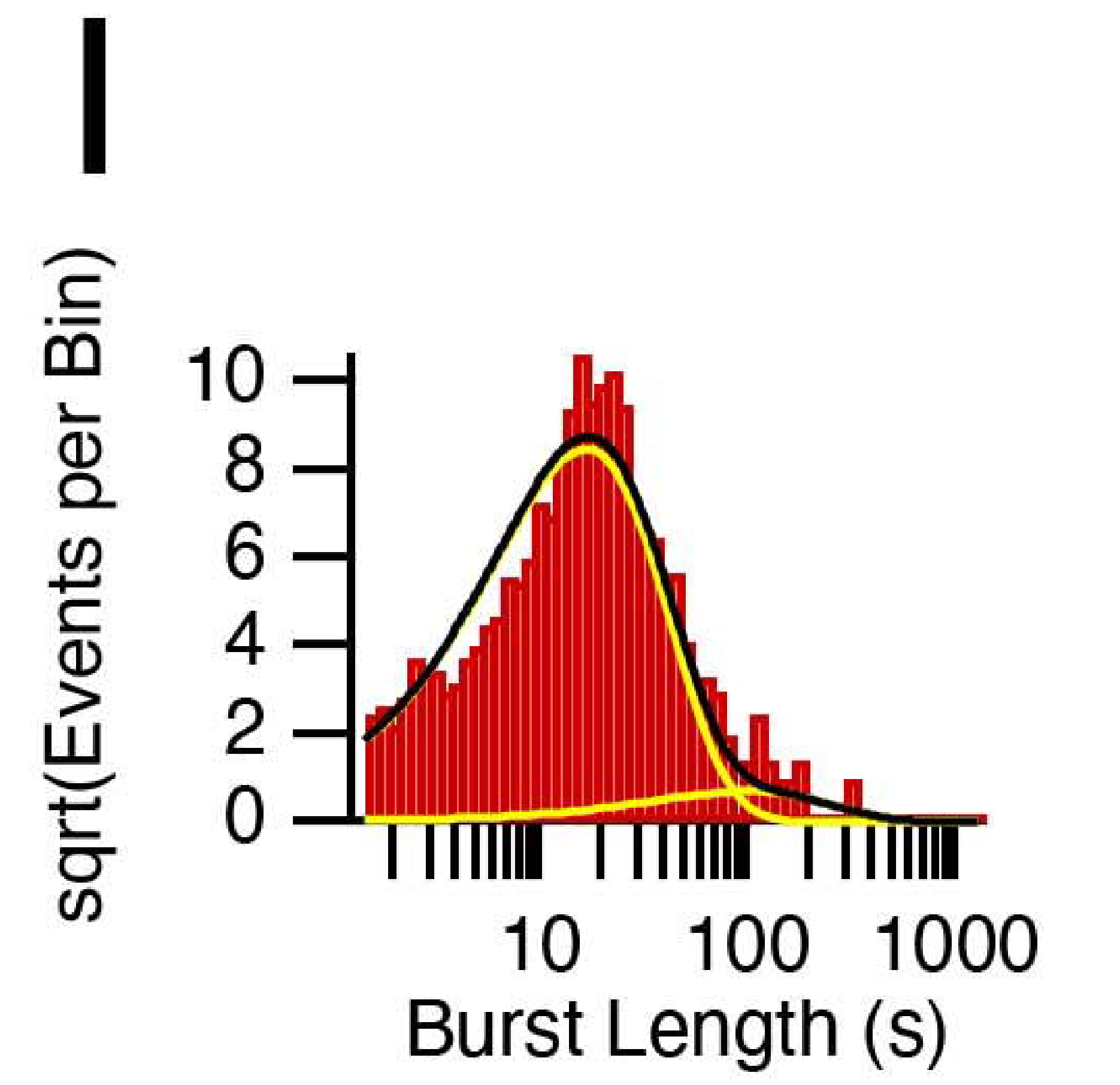

$\mathrm{J}$

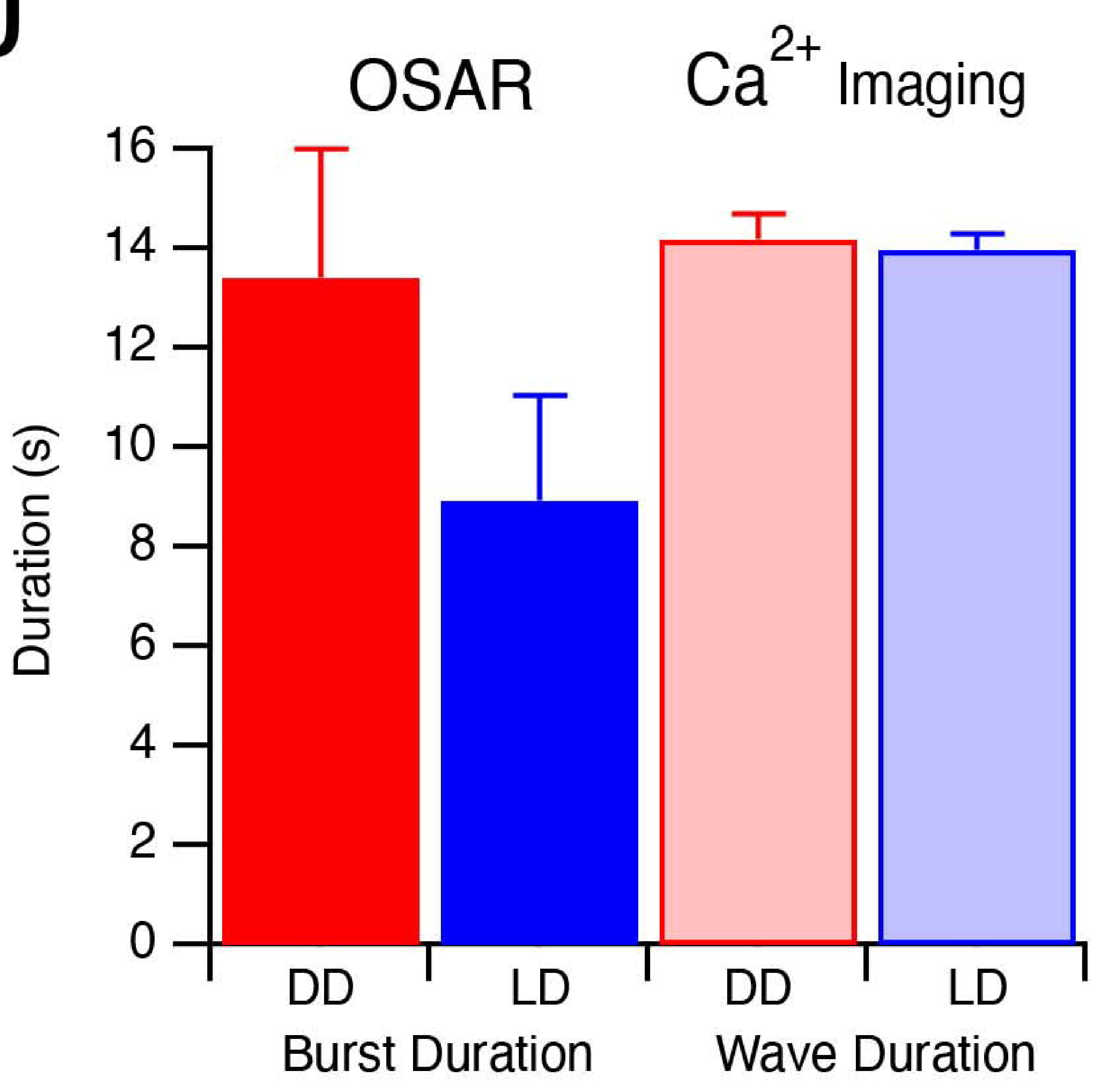


A

Correlation Between Cell 1 and Cell 2
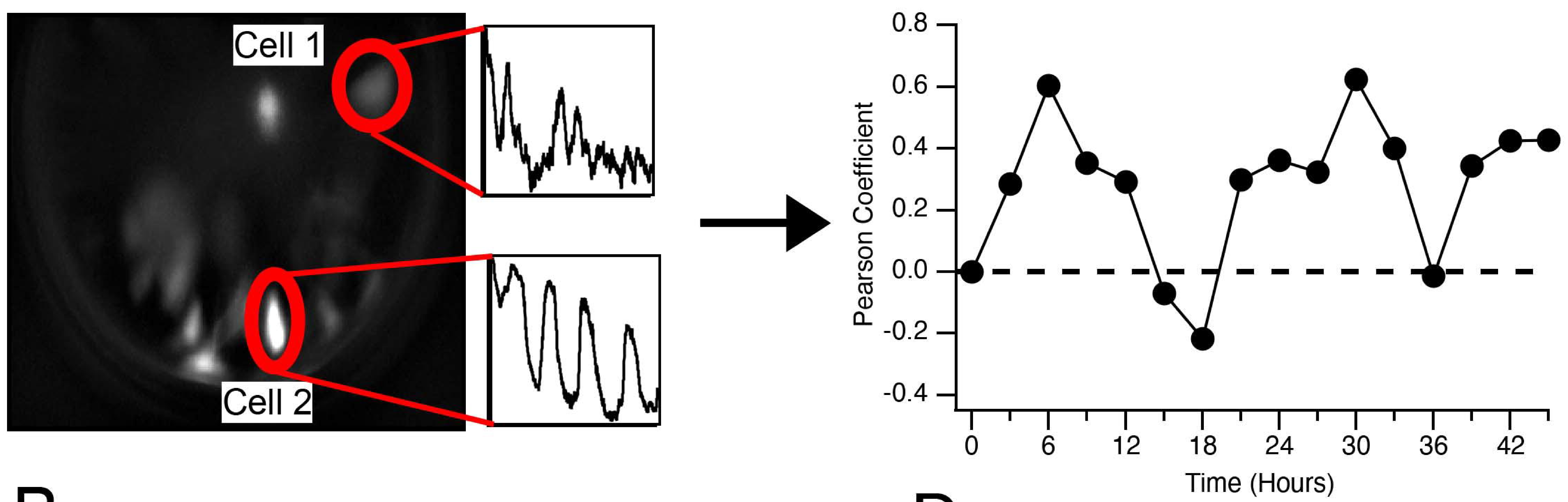

B
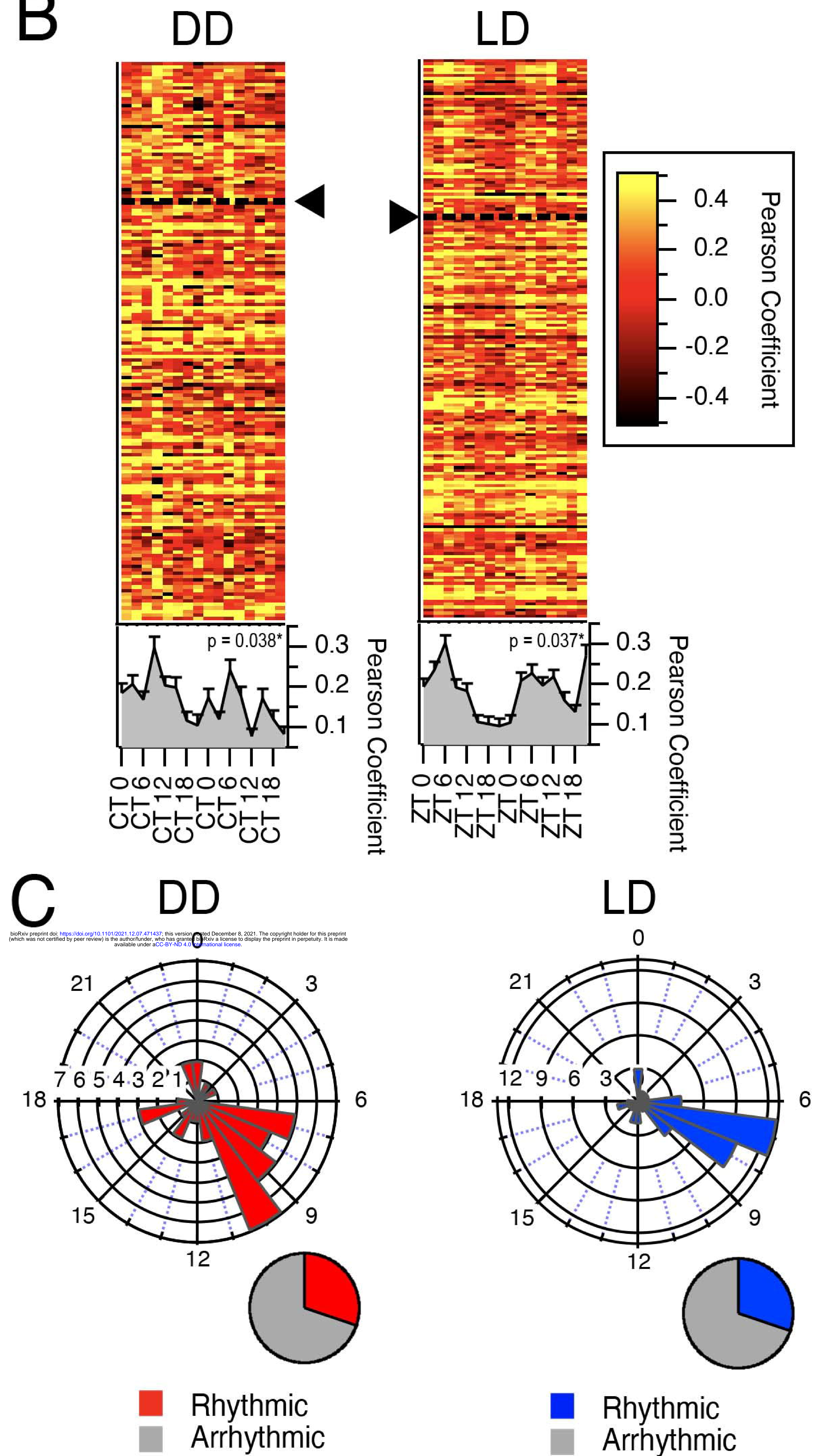

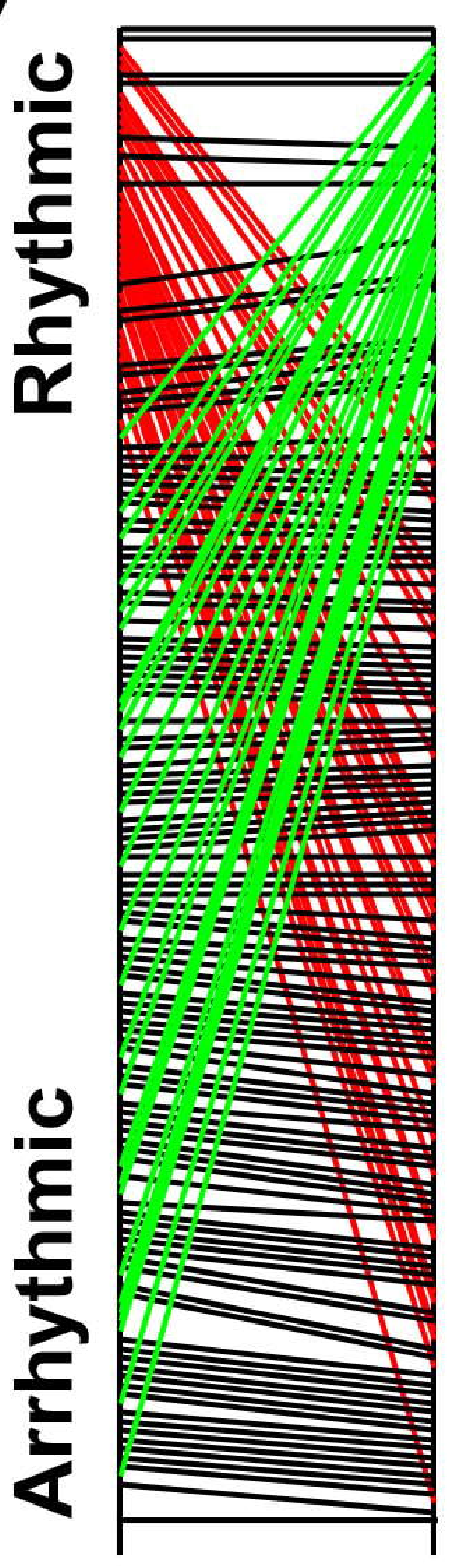

DD1 DD2

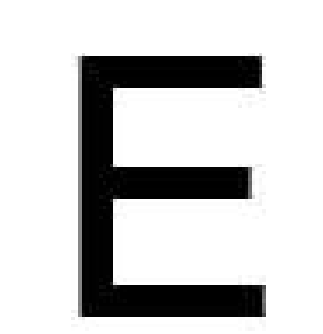

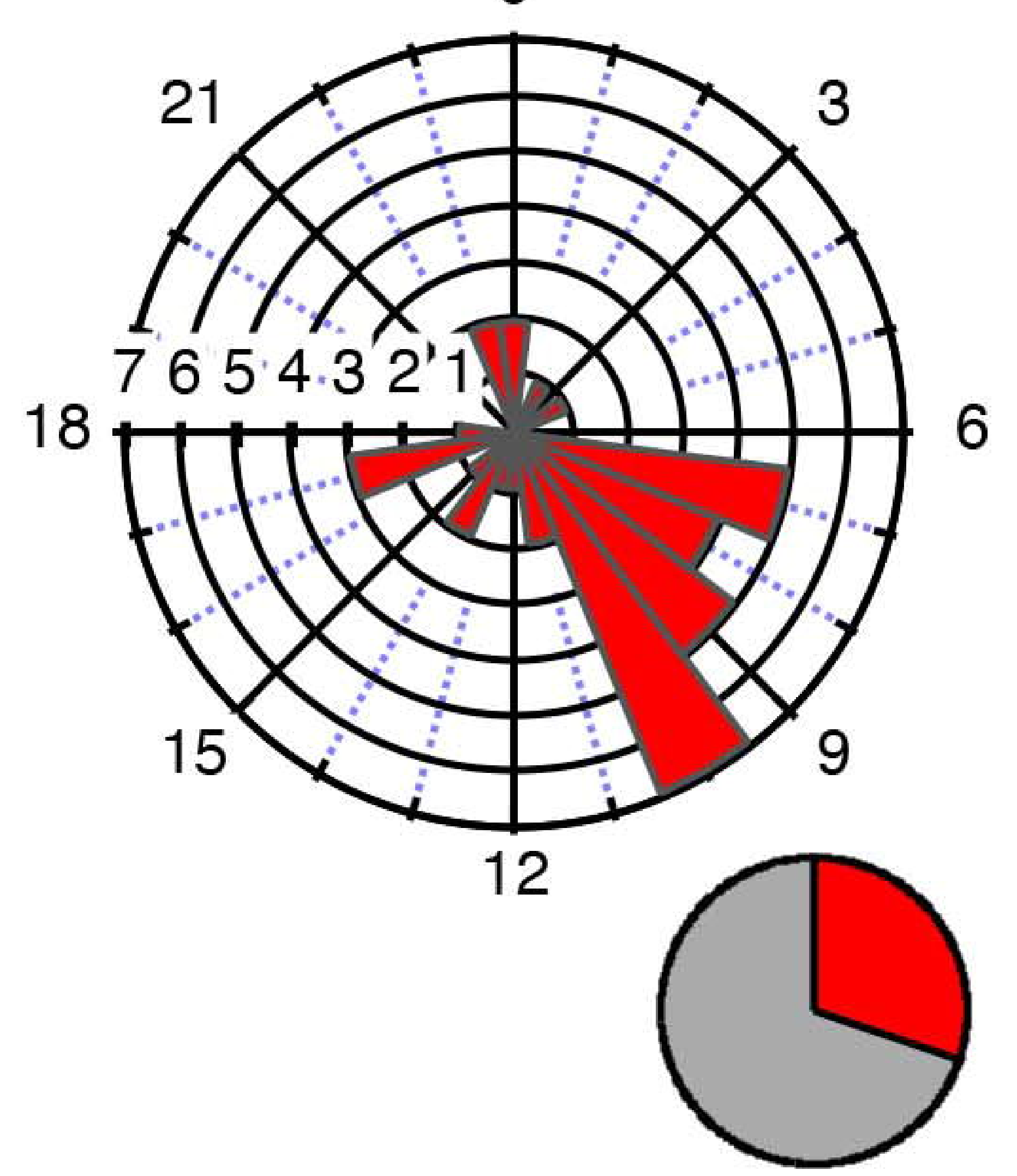

Rhythmic Arrhythmic

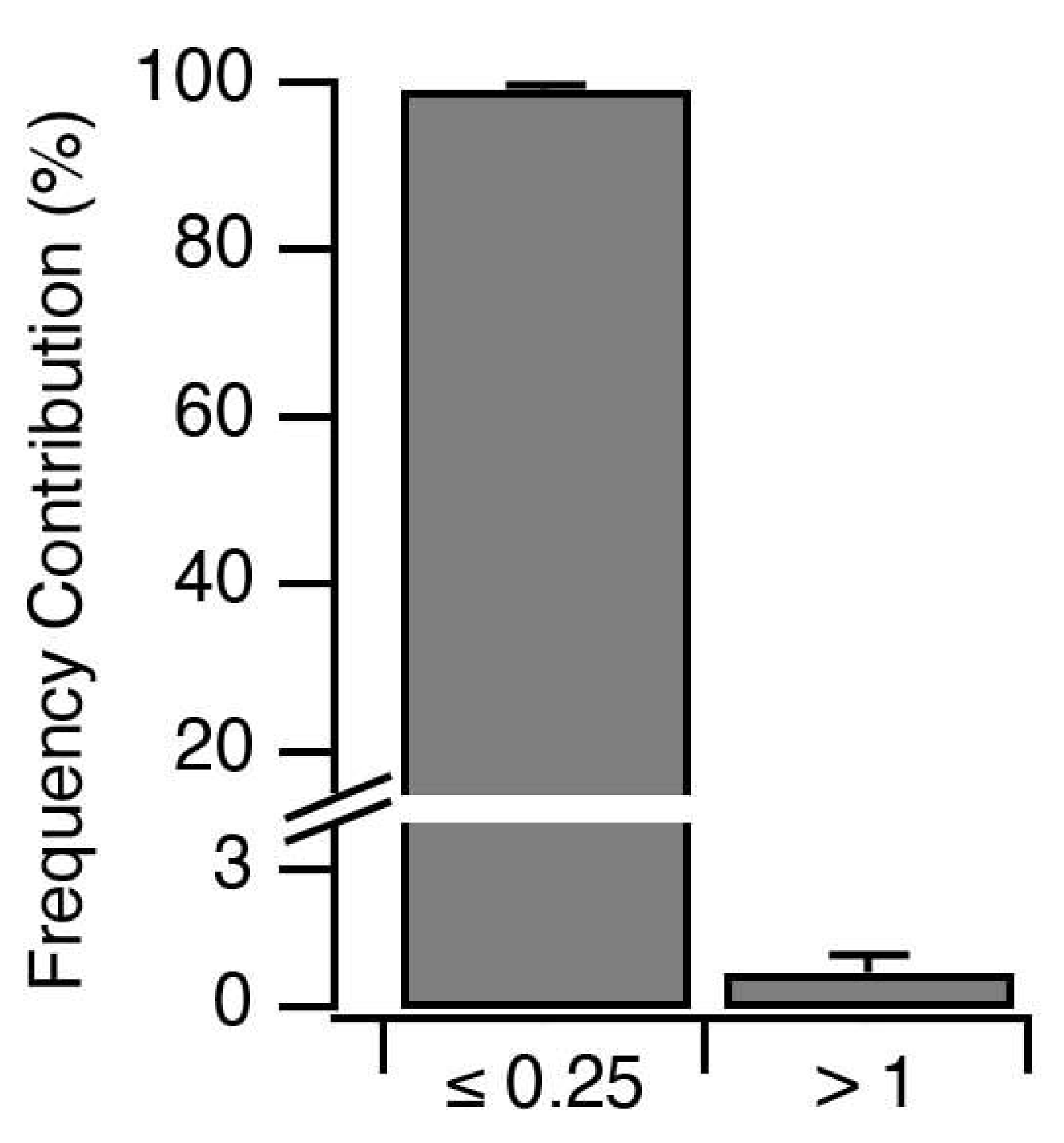

Frequency $(\mathrm{Hz})$ 